\title{
RISK TRADING AND ENDOGENOUS PROBABILITIES IN INVESTMENT EQUILIBRIA*
}

\author{
DANIEL RALPH ${ }^{\dagger}$ AND YVES SMEERS ${ }^{\ddagger}$
}

\begin{abstract}
A risky design equilibrium problem is an equilibrium system that involves $N$ designers who invest in risky assets, such as production plants, evaluate these using convex or coherent risk measures, and also trade financial securities in order to manage their risk. Our main finding is that in a complete risk market - when all uncertainties can be replicated by financial products - a risky design equilibrium problem collapses to what we call a risky design game, i.e., a stochastic Nash game in which the original design agents act as risk neutral and there emerges an additional system risk agent. The system risk agent simultaneously prices risk and determines the probability density used by the other agents for their risk neutral evaluations. This situation is stochastic-endogenous: the probability density used by agents to value uncertain investments is endogenous to the risky design equilibrium problem. This result is most striking when design agents use coherent risk measures in which case the intersection of their risk sets turns out to be a risk set for the system risk agent, thereby extending existing results for risk markets. We also investigate existence of equilibria in both the complete and incomplete cases.
\end{abstract}

Key words. risky design equilibrium problem, risk trading, risky design game, stochasticendogenous equilibrium problem, risk averse, risk measure, complete financial market

AMS subject classifications. 91A15, 91B30, 91B25

DOI. $10.1137 / 110851778$

1. Introduction. We study equilibria over $N$ agents that design or invest in risky assets or stochastic processes representing uncertain future costs (or profits), motivated by risky capital investments such as manufacturing plants. In the style of a Nash game, the $i$ th agent chooses $x_{i}$ from its set of feasible strategies $X_{i} \subset \mathbb{R}^{n}$ knowing the strategies $x_{-i} \in X_{-i} \subset \mathbb{R}^{(N-1) n}$ of other agents, thereby defining its risky cost or risky asset as a random vector $\Xi_{i}\left(x_{i}, x_{-i}\right)$. This risky cost lies in an uncertain outcome space $\mathcal{Z}$ which is either $\mathbb{R}^{K}$ if there are $K$ future scenarios or an $L_{p}$ space, usually with $1 \leq p<\infty$, if there are infinitely many scenarios. We think of $\Xi_{i}\left(x_{i}, x_{-i}\right)$ as the uncertain cost, net of sales revenue, of production in a future market; equivalently $-\Xi_{i}\left(x_{i}, x_{-i}\right)$ represents future profit. In terms of microeconomics, $\Xi_{i}\left(x_{i}, x_{-i}\right)$ is an abstract description of stochastic production sets which relate inputs to outputs.

Given a probability density $\Pi$ on $\mathcal{Z}$, the expectation $\mathbb{E}_{\Pi}\left[\Xi_{i}\left(x_{i}, x_{-i}\right)\right]$ is a risk neutral valuation of agent $i$ 's risky cost. This leads to a risk neutral Nash game,

$$
\min _{x_{i}} \mathbb{E}_{\Pi}\left[\Xi_{i}\left(x_{i}, x_{-i}\right)\right] \quad \text { subject to } \quad x_{i} \in X_{i}
$$

for $i=1, \ldots, N$. Our interest, however, is in agents that are risk averse.

Suppose agent $i$ puts a value of $r_{i}\left(\Xi_{i}\left(x_{i}, x_{-i}\right)\right)$ on its uncertain cost using a convex risk measure [16] denoted $r_{i}: \mathcal{Z} \rightarrow \mathbb{R}$. It can also hedge its risky cost, i.e., change $\Xi_{i}\left(x_{i}, x_{-i}\right)$ to $\Xi_{i}\left(x_{i}, x_{-i}\right)-W_{i}$ by purchasing financial securities or contracts

\footnotetext{
${ }^{*}$ Received by the editors October 17, 2011; accepted for publication (in revised form) July 24, 2015; published electronically December 22, 2015.

http://www.siam.org/journals/siopt/25-4/85177.html

${ }^{\dagger}$ Centre for Risk Studies, Judge Business School, University of Cambridge, Cambridge CB2 1AG, UK (d.ralph@jbs.cam.ac.uk).

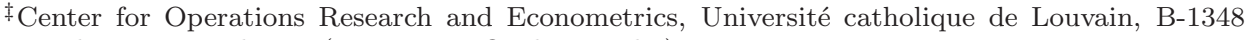
Louvain-la-Neuve, Belgium (yves.smeers@uclouvain.be).
} 
as represented by a vector $W_{i} \in \mathcal{Z}$. Given the price of risk $P^{\mathrm{r}}$ in the dual space of $\mathcal{Z}$, the cost of $W_{i}$ is defined by the dual action $P^{\mathrm{r}}\left[W_{i}\right] \in \mathbb{R}$. Hence agent $i$ optimizes with respect to both design and hedging variables,

$$
\min _{x_{i}, W_{i}} P^{\mathrm{r}}\left[W_{i}\right]+r_{i}\left(\Xi_{i}\left(x_{i}, x_{-i}\right)-W_{i}\right) \quad \text { subject to } \quad x_{i} \in X_{i} .
$$

Recall the classical risk market [1] in which each agent $i$ has a risky cost $Z_{i} \in \mathcal{Z}$, e.g., $Z_{i}=\Xi_{i}\left(x_{i}, x_{-i}\right)$ for fixed $x_{i}$ and $x_{-i}$, and is given the price of risk $P^{\mathrm{r}}$. Agent $i$ hedges $Z_{i}$ by solving

$$
\min _{W_{i}} P^{\mathrm{r}}\left[W_{i}\right]+r_{i}\left(Z_{i}-W_{i}\right) .
$$

The price of risk $P^{\mathrm{r}}$ is determined by the equilibrium condition that all trades of financial products balance each other:

$$
\sum_{i=1}^{N} W_{i}=0
$$

This describes a complete risk market, i.e., when $W_{i}$ can be any member of $\mathcal{Z}$.

DEFINITION 1. The risky design equilibrium problem ${ }^{1}$ is the system that combines (2) (for $i=1, \ldots, N$ ) and (4). A risky design equilibrium is a solution of this system that comprises design variables $x_{\uparrow}$, risk trades $W_{\uparrow}$, and a price of risk $P^{\mathrm{r}}$.

A risky design equilibrium problem combines a risk averse Nash game in design variables $x_{\uparrow}:=\left(x_{i}\right)_{i=1}^{N}$ with a (complete) market in risk. Incomplete risky design equilibrium problems, where risk trades $W_{i}$ are constrained to lie in a closed subspace of $\mathcal{Z}$, are addressed in section 4 .

Our main contribution is to build a bridge between the risky design equilibrium problem (2), (4) and the risk neutral design game (1), which we do by introducing a risky design game as we now describe. The most striking case is when each $r_{i}$ is a coherent risk measure (CRM) [2], hence

$$
r_{i}(Z)=\max \left\{\mathbb{E}_{\Pi}[Z]: \Pi \in \mathcal{D}_{i}\right\}
$$

for some nonempty, closed, convex set of probability densities $\mathcal{D}_{i}$ called its risk set. This is a worst-case appraisal of the cost of $Z$ over all $\Pi \in \mathcal{D}_{i}$. Let $\mathcal{D}_{0}$ be the intersection $\bigcap_{i=1}^{N} \mathcal{D}_{i}$, the system risk set (see Definition 4 in section 2.2.1). Nonemptiness of $\mathcal{D}_{0}$ can be assumed since this is necessary for existence of a risk market equilibrium (see part 2 of Theorem 4, section 2.2.1). We reformulate the risky design equilibrium problem as a game combining the risk neutral design game (1), over $N$ players, with an $N+1^{\text {st }}$ agent, the system risk agent, that sets the probability density $\Pi$ by solving

$$
\max _{\Pi} \mathbb{E}_{\Pi}\left[\sum_{i=1}^{N} \Xi_{i}\left(x_{i}, x_{-i}\right)\right] \quad \text { subject to } \Pi \in \mathcal{D}_{0} .
$$

That is, the system risk agent evaluates the system $C R M$ whose risk set is $\mathcal{D}_{0}$.

The combination of (1) and (5) exemplifies the risky design game mentioned above. See Definition 5 in section 3.3 for the more general case when agents use convex risk measures.

\footnotetext{
${ }^{1}$ We make a distinction between an equilibrium problem and a game in that, in the former, the price is not provided by a preexisting agent (that optimizes its utility) but emerges from the market as a whole.
} 
Our main finding is that a risky design equilibrium $x_{\uparrow}, W_{\uparrow}, P^{\mathrm{r}}$ yields a solution $x_{\uparrow}, \Pi=P^{\mathrm{r}}$ of the risky design game, and the converse holds under mild technical conditions. This is explored in detail in section 3, including existence results; see Theorem 8 and Corollary 2.

The leap described from risky design equilibria to risky design games and back is considerable. It entails that the probability density $\Pi$ is both the price of risk and the worst-case probability density for every design agent's hedged cost $\Xi_{i}\left(x_{i}, x_{-i}\right)-W_{i}$. That is, at equilibrium, the marginal view of risk (which turns out to be a probability density) is the same for all design agents, which is why they appear as risk neutral in the risky design game. Since $\Pi$ is part of the solution of this system, we say that the equilibrium problem is stochastic-endogenous.

The properties of $\Pi$, above, extend important work in the finance literature $[1,4$, $7,8,10,14,15,16,18]$, in which agents are endowed with risky assets that they value with risk measures, and trade financial securities to manage risk. This is reviewed in section 2. The underlying theory of risk measures $[2,16]$ is also an expanding topic in stochastic optimization theory [31,33]. The design equilibrium setting combines both topics by modeling competition between risk averse agents that invest in both risky assets and financial securities.

Section 4 concludes the paper with a short exploration of risky design equilibria when the risk market is incomplete, namely, each risk trade $W_{i}$ is confined to a closed subspace $\mathcal{W}$ of $\mathcal{Z}$. Once again we reformulate the equilibrium problem as a hybrid of a risk neutral Nash game and a risk pricing equilibrium problem; see Theorem 11. This diverges from the complete case in that, at equilibrium, each agent may use a different probability density (or price of risk) to describe its marginal view of risk. Although we are unable to convert this hybrid equilibrium problem to a Nash game, we use Kakutani's fixed point theorem to provide existence of equilibria; see Theorem 12.

Before proceeding to the main sections of the paper we give a literature review in section 1.1 and set up our notation in section 1.2.

1.1. Motivation and the broader literature. In this paper the stochastic process $\Xi_{i}\left(x_{i}, x_{-i}\right)$ is generic. For example, in the robust competitive newsvendor setting [20] it would be cost net of uncertain sales, where a quantity $x_{i}$ of newspapers is ordered today for sales tomorrow and the level of uncertain demand to be serviced by newsvendor $i$ depends on others' orders $x_{-i}$.

In fact our motivation originates from our interest in restructured electricity systems. Restructuring induced a move from the optimization to the equilibrium paradigm in order to represent the change of structure of the industry. These equilibrium models are often of the stochastic programming type but make two simplifications. First, the underlying stochastic processes (e.g., of prices) are exogenous and do not depend on the decisions embedded in the model; second, the models are often formulated in a risk neutral world without any representation of hedging processes. Both simplifications depart from the reality of the restructured electricity system and should be remedied. Our model is a step in this direction: it contains financial hedging instruments that are priced endogenously.

Richer models involving two-stage or multistage operational or investment decisions underlie the abstract setting we develop. The electricity investment model proposed in [11] is of this type and was one of the inspirations for the work presented here. Our paper, or rather the first version of it [26], is cited in work on two-stage capacity equilibria [12] and two-stage hydropower equilibria [25]. It is also cited in [22], which combines a market in financial instruments with a spot electricity market, 
so that payoffs are endogenous, and considers an incomplete risk market that yields a generalized Nash equilibrium.

While our model is an extension of partial equilibrium models found in the literature, it can also be seen as a simplification of the generalization of general equilibrium models to stochastic models of physical and financial assets. An in-depth analysis of the economic work in that area can be found in [23]. The paper [21] presents a mathematical programming-based analysis of these questions. Our model works in a simpler context but it may be useful to briefly note some relations between the two areas. In contrast with stochastic general equilibrium (SGE) problems, we neglect the consumer side because of the complexity in SGE problems introduced by two-stage budget constraints. We also state our problem in a Nash equilibrium set-up which avoids the complication of pricing physical assets (we only deal with the prices of the financial assets). Our model is thus a pure production model; an extension that embeds a consumer with constant marginal utility of money (hence still avoiding the complexity of the intertemporal budget constraint) and explicitly deals with the prices of the physical assets is in preparation.

Although our main results pertain to complete markets, a treatment of incomplete markets, as in the SGE case, is closer to reality. In an incomplete market the space of revenue from the physical market cannot be spanned by the financial products (which is typical in electricity markets) and hence where a standard nonarbitrage condition leads to a multiplicity of prices. In the language of SGE models [23], we consider equilibrium in "constrained equilibrium allocation," i.e., in the space of financial transfers spanned by existing instruments, and show existence of an equilibrium in terms of risk adjusted evaluation of profits, taking into account the variety of prices. A final note is that although Pareto optimality is an ever-present topic in economics, from welfare theory through to recent work on financial markets, the question of Pareto optimality for these equilibria is left for further research.

\subsection{Notation.}

Simple finite dimensional case. Let $\mathbb{R}$ denote the set of real numbers, $\mathbb{R}_{+}:=$ $[0, \infty), \mathbb{R}_{\infty}:=\mathbb{R} \cup\{\infty\}$. We will identify each future scenario or state $\omega$ with a future cost, e.g., $Z_{\omega} \in \mathbb{R}$, and also identify probability density functions on the set $\Omega$ of all scenarios. For example, if $\Omega=\{1, \ldots, K\}$, then $\mathcal{Z}=\mathbb{R}^{K}$, so that an uncertain outcome $Z \in \mathcal{Z}$ represents $K$ cost scenarios, and the set of probability densities $\mathcal{P}$ is defined as the set of vectors in $\mathbb{R}^{K}$ with nonnegative entries that sum to 1 .

The usual dot or inner product between any vectors $\zeta$ and $Z$ in $\mathcal{Z}$ is $\zeta[Z]:=$ $\sum_{\omega=1}^{K} \zeta_{\omega} Z_{\omega}$. We also introduce $\mathbb{1}$ as the vector of ones in $\mathcal{Z}$, and the comparison $Z_{1} \preceq Z_{2}$ between vectors in $\mathcal{Z}$ to mean that $Z_{1 \omega} \leq Z_{2 \omega}$ for each $\omega$. This allows us to write $\mathcal{P}$ in dual form as those $\zeta \in \mathbb{R}^{K}$ such that $\zeta[Z] \geq 0$ for all $Z \succeq 0$ and $\zeta[\mathbb{1}]=1$. We usually denote a probability density by $\Pi$ and write its dual action as an expectation: $\mathbb{E}_{\Pi}[Z]:=\Pi[Z]$.

We write a list of uncertain outcomes, one for each agent, as $Z_{\uparrow}:=\left(Z_{i}\right)_{i=1}^{N}$. Thus $Z_{\uparrow}$ belongs to $\mathcal{Z}^{N}$, which is the Cartesian product of $N$ copies of $\mathcal{Z}$. Likewise, when we introduce financial products in section 2.1.2, we will write $W_{\uparrow}:=\left(W_{i}\right)_{i=1}^{N} \in \mathcal{Z}^{N}$.

For $x, v \in \mathbb{R}^{n}$, the inner product is denoted $v^{\top} x$ (we use different notation from that of the inner product on $\mathcal{Z}$, to avoid confusing $\mathbb{R}^{n}$ with $\mathcal{Z}$.) For a set $D \subset \mathbb{R}^{n}$ recall that its tangent cone at a point $x \in D$, written $T_{D}(x)$, is the set of points of the form $\lim \left(x_{\nu}-x\right) / t_{\nu}$, where $\left\{x_{\nu}\right\} \subset D$ converges to $x$ and $0<t_{\nu} \rightarrow 0$. If $D$ is convex and $x \in D$, then $T_{D}(x)$ is nonempty, closed and convex; and both $D$ and $T_{D}(x)$ have nonempty relative interior [29]. 
For readers whose primary interest is in stochastic situations with finitely many scenarios, the remainder of this section can be skipped. We suggest reading subsequent results with $K$ scenarios in mind, $\mathcal{Z}$ and its dual space $\mathcal{Z}^{*}$ as $\mathbb{R}^{K}$, and other notation as above.

$\boldsymbol{L}_{p}$ spaces and uncertain outcome spaces. To allow for an infinite number of scenarios we need more notational tools. Adapting [31], let $(\Omega, \mathcal{F}, \mu)$ be a measure space that is nonnegative, nontrivial, and finite, i.e., the measure of sets $S$ in $\mathcal{F}$ satisfies $\mu(S) \geq 0$ and, in particular, $0<\mu(\Omega)<\infty$. $^{2}$ (The prototype such measure space is the real interval $[0,1]$ under Lebesgue measure.) Henceforth measurable means $\mathcal{F}$-measurable, and the measure of a set is taken with respect to $\mu$.

For $1 \leq p<\infty$ let $L_{p}(\Omega, \mathcal{F}, \mu)$, or $L_{p}(\Omega)$ for short, be the space of measurable functions $^{3} f: \Omega \rightarrow \mathbb{R}$ with finite $p$-norm, defined as $\|f\|_{p}:=\left(\int_{\Omega}|f(\omega)|^{p} d \mu(\omega)\right)^{1 / p}$. The space $L_{\infty}(\Omega, \mathcal{F}, \mu)$ is defined similarly with $\|f\|_{\infty}:=\inf \left\{\sup _{\omega \in \Omega \backslash S}|f(\omega)|\right.$ : $S$ has measure zero in $\Omega\}$. We usually emphasize the vector view by referring to members of $L_{p}(\Omega, \mathcal{F}, \mu)$ in the form $Z=\left(Z_{\omega}\right)_{\omega \in \Omega}$ rather than as functions on $\Omega$ and may write $\|Z\|$ rather than $\|Z\|_{p}$ when the context is clear. These $L_{p}$ spaces are Banach spaces that, as a consequence of the finite measure of $\Omega$, are contained in $L_{1}(\Omega, \mathcal{F}, \mu)$.

DEFINITION 2 (uncertain outcome spaces). $\mathcal{Z}$ is an uncertain outcome space if it is either $\mathbb{R}^{K}$ or $L_{p}(\Omega)$ for some $1 \leq p \leq \infty$, where $(\Omega, \mathcal{F}, \mu)$ is a nonnegative, nontrivial, finite measure space. Each $Z$ in $\mathcal{Z}$ may be called an uncertain outcome or uncertain vector or random vector.

More general spaces of uncertain outcomes are discussed in [31, 33]. Nevertheless $L_{p}$ spaces are quite rich and we prefer ease of presentation to greater generality.

There is a natural ordering of uncertain outcomes $Z_{1}, Z_{2} \in \mathcal{Z}: Z_{1} \preceq Z_{2}$ denotes $Z_{1 \omega} \leq Z_{2 \omega}$ for almost all (a.a.) $\omega \in \Omega$, where a.a. $\omega$ means either all $\omega \in \Omega$ if $\Omega=\{1, \ldots, K\}$ or all $\omega$ except those in a measure zero subset of $\Omega$ if $\Omega$ is a more general measure space. Likewise a.e. means for a.a. $\omega$.

The dual space $\mathcal{Z}^{*}$ and set of probability densities $\mathcal{P}$. The topological dual $\mathcal{Z}^{*}$ of $\mathcal{Z}=L_{p}(\Omega, \mathcal{F}, \mu)$ is conveniently expressed if $1 \leq p<\infty$. By choosing $q$ to satisfy $(1 / p)+(1 / q)=1$ it is well known that $1<q \leq \infty$ and $\mathcal{Z}^{*}=L^{q}(\Omega, \mathcal{F}, \mu)$, where the dual action of $g \in L^{q}(\Omega, \mathcal{F}, \mu)$ on $f \in L_{p}(\Omega, \mathcal{F}, \mu)$ is $g[f]:=\int_{\Omega} f(\omega) g(\omega) d \mu(\omega)$. Analogous to probability densities on $\mathbb{R}^{K}$, we define $\mathcal{P}$ as the set of dual elements $g$ with $^{4} g \succeq 0$ and $g[\mathbb{1}]=\int_{0}^{1} g(\omega) d \mu(\omega)=1$, and observe that the uniform distribution $\mathbb{1} / \mu(\Omega)$ lies in $\mathcal{P}$ when $\mathcal{Z}$ is an $L_{p}$ space.

2. The toolbox of risk markets and risk measures. Here we gather and recast results from a number of papers in the finance literature, including $[1,4,7$, $8,9,10,14,15,16,18]$, to provide tools for complete and perfectly competitive risk markets; these and other papers will be highlighted in the material to follow.

Section 2.1 introduces risk markets, where agents use convex functions to assess uncertainty and trade financial products to hedge risk. The important results for us

\footnotetext{
${ }^{2}$ Without loss of generality the measure of $\Omega$ can be normalized to 1 , making $(\Omega, \mathcal{F}, \mu)$ a probability measure space as in [31]. We avoid this to make the analogy between $\mathbb{R}^{K}$ and $L_{p}$ spaces more direct.

${ }^{3}$ Actually elements of $L_{p}$ are classes of measurable functions that are equal up to sets of measure zero, which is appropriate for Banach space theory. We will find it more convenient to refer to specific functions, or their vector forms, to designate their equivalence classes.

${ }^{4}$ More generally, $g \succeq 0$ if and only if $g[f] \geq 0$ for each $f \in L_{p}(\Omega, \mathcal{F}, \mu)$ with $f \succeq 0$.
} 
are that the existence of a risk (market) equilibrium is equivalent to solving a system risk optimization problem that follows as (6); that the optimal value of this system risk problem can be written as an inf-convolution $v^{\mathrm{r}}$, defined by $(7)$, that we call the system risk function; and, when a risk equilibrium price $P^{\mathrm{r}}$ exists, that the price of risk is characterized as a subgradient of $v^{\mathrm{r}}$.

Section 2.2 specializes risk markets to the case of (convex) risk measures and CRMs. An important first result is that the subgradients of these functions are probability densities. Next, when each $r_{i}$ is a risk measure, the system risk function $v^{\mathrm{r}}$ is also a risk measure; hence, when a risk equilibrium exists, $P^{\mathrm{r}}$ is a probability density. The situation is particularly striking when each $r_{i}$ is a CRM, for then $v^{\mathrm{r}}$ is a CRM whose risk set can be characterized as the intersection of the risk sets of all $N$ agents.

The toolbox is concluded in section 2.3, which presents various sufficient conditions for existence of risk equilibria.

\subsection{Introduction to a market for risk.}

2.1.1. Convex functions for assessing uncertain outcomes. Suppose there are $N$ agents indexed by $i$ that are endowed with respective uncertain assets $Z_{i} \in \mathcal{Z}$. Agent $i$ has its own risk function, $r_{i}: Z \rightarrow \mathbb{R}$, that is convex. (In section 2.2 we will introduce further axioms to define risk measures.) Agent $i$ knows each payoff $Z_{i \omega}$ with certainty, but is not sure which of these cost outcomes, i.e., which scenario $\omega$, will occur in the future. Agents want to minimize cost or, equivalently, maximize profit, which is taken to be synonymous with negative cost.

Consider a continuous convex function $r: \mathcal{Z} \rightarrow \mathbb{R}$ where $\mathcal{Z}$ is a Banach space. Note that $r$ is subdifferentiable at any $Z \in \mathcal{Z}$, the latter meaning that the subdifferential defined below is nonempty:

$$
\partial r(Z):=\left\{\zeta \in \mathcal{Z}^{*}: r(Z)+\zeta[Y-Z] \leq r(Y) \text { for all } Y \in \mathcal{Z}\right\} .
$$

Moreover, the subdifferential is also locally bounded near any $Z \in \mathcal{Z}$; these results, which we will use without reference, can be found in [30, Theorems 8 and 11(a)]. It is elementary that $z$ is a local or equivalently a global minimum of $\min _{Z \in \mathcal{Z}} r(Z)$ if and only if the optimality condition $0 \in \partial r(Z)$ holds.

2.1.2. A complete and perfectly competitive risk market. For $i=1, \ldots$, $N$ let us fix $Z_{i} \in \mathcal{Z}$ as agent $i$ 's endowment and write the list of endowments as $Z_{\uparrow}=\left(Z_{i}\right)_{i=1}^{N} \in \mathcal{Z}^{N}$. Recall the risk market given by (3) for each $W_{i}$ and (4) for the $P^{\mathrm{r}}$.

Our first result, essentially due to [1], states that the equivalence between perfectly competitive trading and system optimization "is a trivial reformulation of the usual one in welfare economics" given by [32, Chapter VIII]. This adapts the classical paradigm of perfect competition from goods and services to risk, i.e., each agent sets its consumption level (of financial products $W_{i}$ ) to balance its marginal risk reduction against the unit prices $\left(P^{\mathrm{r}}\right)$ of those products.

Theorem 1. Let $\mathcal{Z}$ be a Banach space, $r_{i}: \mathcal{Z} \rightarrow \mathbb{R}$ be convex and lower semicontinuous for $i=1, \ldots, N$, and the list of agents' endowments, $Z_{\uparrow}=\left(Z_{i}\right)_{i=1}^{N} \in \mathcal{Z}^{N}$, be given. The following are equivalent:

1. There exists a market equilibrium for risk, i.e., a price vector $P^{\mathrm{r}} \in \mathcal{Z}^{*}$ and a balanced list of risk trades $W_{\uparrow}=\left(W_{i}\right)_{i=1}^{N} \in \mathcal{Z}^{N}$ such that $W_{i}$ solves (3) for each $i$. 
2. $W_{\uparrow}$ solves system risk problem

$$
\min _{W_{\uparrow}} \sum_{i=1}^{N} r_{i}\left(Z_{i}-W_{i}\right) \quad \text { subject to } \quad \sum_{i=1}^{N} W_{i}=0
$$

and $P^{\mathrm{r}}$ is the Lagrange multiplier of trade balance constraint.

3. $W_{\uparrow}$ is balanced, $\bigcap_{i=1}^{N} \partial r_{i}\left(Z_{i}-W_{i}\right)$ is nonempty, and $P^{\mathrm{r}}$ is a member of this intersection.

The equivalence between statements 1 and 2 is classical economics [32] and is immediately derived by examining the stationary conditions [30] for the convex optimization problems that are involved. The equivalence between statements 1 and 3 is elementary and also follows easily from stationary conditions without resorting to Lagrange multipliers (or checking a regularity condition on the constraints) by defining $W_{N}=-\sum_{i=1}^{N-1} W_{i}$ in order to rewrite (6) as an unconstrained optimization problem over $W_{1}, \ldots, W_{N-1}$.

We introduce the system risk function of the consolidated or aggregate portfolio $Z_{0}=\sum_{i} Z_{i}$

$$
v^{\mathrm{r}}\left(Z_{0}\right):=\inf \left\{\sum_{i=1}^{N} r_{i}\left(Z_{i}\right): Z_{\uparrow} \in \mathcal{Z}^{N}, \sum_{i=1}^{N} Z_{i}=Z_{0}\right\} \quad \text { for all } Z_{0} \in \mathcal{Z} .
$$

This is a classical inf convolution of the functions $r_{i}$ and is therefore convex when each $r_{i}$ is convex [29]. Note that $v^{\mathrm{r}}\left(\sum_{i} Z_{i}\right)$ is the infimal value (or optimal value if a solution exists) of (6). The theory of inf convolutions is used later in section 2.3 when discussing existence of a solution to (7).

Our next result is extracted and restated from [10].

TheOREM 2 (see [10, section 3.2]). Under the assumptions of Theorem 1, if a risk market equilibrium exists for a given list $Z_{\uparrow} \in \mathcal{Z}^{N}$ of agents' endowments, then the following are equivalent:

1. $P^{\mathrm{r}}$ prices risk.

2. $P^{\mathrm{r}}$ lies in $\partial v^{\mathrm{r}}\left(\sum_{i=1}^{N} Z_{i}\right)$.

2.2. Introduction to risk measures. Recall the notation $Z_{1} \preceq Z_{2}$ which means $Z_{1 \omega} \leq Z_{2 \omega}$ a.a. $\omega$.

DeFINITION 3 (risk measures). Let $\mathcal{Z}$ be an uncertain outcome space, $r: \mathcal{Z} \rightarrow \mathbb{R}$, and consider the following properties:

Axiom 1. Convexity.

Axiom 2. Monotonicity: $r\left(Z_{1}\right) \leq r\left(Z_{2}\right)$ for $Z_{1}, Z_{2} \in \mathcal{Z}$ with $Z_{1} \preceq Z_{2}$.

Axiom 3. Translation invariance: $r(Z+\alpha \mathbb{1})=r(Z)+\alpha$ for $Z \in \mathcal{Z}$ and $\alpha \in \mathbb{R}$.

Axiom 4. Positive homogeneity: $\rho(\alpha Z)=\alpha \rho(Z)$ for $Z \in \mathcal{Z}$ and $\alpha>0$.

We say $r$ is a (convex) risk measure [16] if it satisfies axioms 1-3 and is a CRM [2] if it additionally satisfies axiom 4.

We note that $[5,6,31,33]$ all provide bridges between the finance and optimization approaches to risk measures though the optimized certainty equivalent of [5] was defined prior to the coining of the terms convex and CRMs.

We list some properties of risk measures that we will use without reference below.

TheOREM 3. Let $\mathcal{Z}$ be an uncertain outcome space and $r: \mathcal{Z} \rightarrow \mathbb{R}$.

1. [31, Proposition 3.1] If $r$ is a risk measure, then it is continuous.

2. [16] $r$ is a risk measure if and only if it is convex and its subgradients are probability densities.

Copyright (C) by SIAM. Unauthorized reproduction of this article is prohibited. 
3. [2] $r$ is a CRM if and only if for some nonempty, closed, and convex set $\mathcal{D}$ of probability densities we have $r(Z)=\max _{\zeta \in \mathcal{D}} \zeta[Z]$.

The closed convex set $\mathcal{D}$ associated with the $\mathrm{CRM} \max _{\Pi \in \mathcal{D}} \mathbb{E}_{\Pi}[\cdot]$ is called its risk set.

2.2.1. Pricing risk under risk measures. In this section we combine sections 2.1 and 2.2 to analyze markets for risk when agents are risk averse and express their value of future, uncertain outcomes via risk measures or CRMs. Risk markets exemplify equilibria under risk. More complex situations that combine risk trading with design or investment decisions are discussed in sections 3 and 4 .

We study $N$ agents (e.g., investors in production plants) where agent $i$ is endowed with an uncertain cost vector $Z_{i}=\left(Z_{i \omega}\right)_{\omega \in \Omega}$ (e.g., the cost of production tomorrow), and each $\omega$ indexes a future scenario (e.g., relating to cost of inputs or price of output). Agents are risk averse and exchange financial securities to reduce their risk.

We introduce a CRM to characterize system risk when all agents use CRMs.

Definition 4 (system CRM). Let $\mathcal{Z}$ be an uncertain outcome space. For each agent $i=1, \ldots, N$, let $r_{i}: \mathcal{Z} \rightarrow \mathbb{R}$ be a CRM and denote its (nonempty, convex, and closed) risk set as $\mathcal{D}_{i} \subset \mathcal{P}$. Define the system risk set as

$$
\mathcal{D}_{0}=\bigcap_{i=1}^{N} \mathcal{D}_{i}
$$

and the system CRM as the CRM with the risk set $\mathcal{D}_{0}$ :

$$
r_{0}=\sigma_{\mathcal{D}_{0}} .
$$

We say $r_{0}$ describes the risk aversion of a system agent that is the least risk averse or most risk neutral of all agents. The term "least risk averse" reflects the weakly lower cost of uncertainty faced by the system agent than by any other agent due to the containment of $\mathcal{D}_{0}$ in each $\mathcal{D}_{i}$, hence the inequality $r_{0}[Z] \leq r_{i}[Z]$ for any random outcome $Z$.

The next result shows that the system risk function inherits the risk measure properties of the individual risk functions $r_{i}$. Part 2 is freely adapted from the references cited there.

TheOREM 4. Assume $\mathcal{Z}$ is an uncertain outcome space; there are $N$ agents where each agent $i$ assesses the value of uncertain assets using a risk measure $r_{i}: \mathcal{Z} \rightarrow \mathbb{R}$; and the risk market is perfectly competitive and complete. If agents' endowments are given by $Z_{\uparrow} \in \mathcal{Z}^{N}$ and a corresponding risk market equilibrium exists, then

1. [4, Theorem 3.2], [10, section 3.2] the system risk function $v^{\mathrm{r}},(7)$, is a real valued (convex) risk measure;

2. [7, Propositions 2.5 and 2.7], [8, Lemma 4.3] in the situation of Definition 4, $\mathcal{D}_{0}$ is nonempty and $v^{\mathrm{r}}$ coincides with $r_{0}$ which is the CRM with risk set $\mathcal{D}_{0}$.

2.3. Existence of risk equilibria under risk measures. We give some conditions under which risk can be priced. The case of risk measures is treated in Theorem 5 , followed by a brief review of some important related results in the financial mathematics literature. The case of existence under CRMs follows immediately as Corollary 1.

We recall two well known classes of extended real valued convex functions $f$ : $\mathcal{Z} \rightarrow \mathbb{R}_{\infty}$. We say $f$ is polyhedral convex if its epigraph is the intersection of finitely many closed half spaces in $\mathcal{Z} \times \mathbb{R}$. We say $f$ is law invariant if $f\left(Z_{1}\right)=f\left(Z_{2}\right)$ when $Z_{1}$ 
and $Z_{2}$ are identically distributed on the measure space $(\Omega, \mathcal{F}, \mu)$, written $Z_{1} \sim Z_{2}$, i.e., $\mu\left\{\omega: Z_{1 \omega} \leq \alpha\right\}=\mu\left\{\omega: Z_{2 \omega} \leq \alpha\right\}$ for any $\alpha \in \mathbb{R}$.

We'll need some additional notation. First, the convex conjugate of $f: \mathcal{Z} \rightarrow \mathbb{R}_{\infty}$ is the function $f^{*}: \mathcal{Z}^{*} \rightarrow \mathbb{R} \cup\{\infty\}$ defined by

$$
f^{*}(\zeta):=\sup _{Z \in \mathcal{Z}} \zeta[Z]-v^{\mathrm{r}}(Z) .
$$

Second, the domain of $f^{*}$ is $\operatorname{dom} f^{*}:=\left\{\zeta \in \mathcal{Z}^{*}: f^{*}(\zeta)<\infty\right\}$. Third, the strong quasi-relative interior of a set $S$ in $\mathcal{Z}^{*}$, sqri $(S)$, is the set of $\zeta_{0} \in S$ such that the cone $\left\{\alpha\left(\zeta-\zeta_{0}\right): \alpha>0, \zeta \in S\right\}$ is a closed subspace of $\mathcal{Z}^{*}$.

THEOREM 5. Under the assumptions of Theorem 4, each of the following conditions is sufficient for existence of a risk market equilibrium:

1. Each $r_{i}$ is polyhedral convex and $\bigcap_{i} \operatorname{dom} r_{i}^{*}$ is nonempty.

2. $\mathcal{Z}$ is reflexive, e.g., either $\mathbb{R}^{K}$ or an $L_{p}$ space for $1<p<\infty$, and $\bigcap_{i}$ sqri $\left(\operatorname{dom} r_{i}^{*}\right)$ is nonempty.

3. [15, Theorem 2.5] $\mathcal{Z}$ is either $\mathbb{R}^{K}$ or a nonatomic $L_{p}$ space for $1 \leq p \leq \infty$, each $r_{i}$ is law invariant, and $\bigcap_{i} \operatorname{dom} r_{i}^{*}$ is nonempty.

Sufficiency of statement 1 for existence of a risk equilibrium appears to be new. It relies on the property of a linear program - in this case a reformulation of (6) - that the problem has a solution when it is feasible and bounded below.

The sqri condition in statement 2 is motivated by [18], which studies risk markets with finitely many contingencies $\left(\mathcal{Z}=\mathbb{R}^{K}\right)$ and, hence, uses the equivalent notion of relative interior, rint [29], rather than sqri. Nonemptiness of $\bigcap_{i} \operatorname{rint}\left(\operatorname{dom} r_{i}^{*}\right)$ is shown [18, Proposition 4.2] to be equivalent to the existence of risk trades $W_{\uparrow}$ that are balanced such that the adjusted list of assets $Z_{\uparrow}-W_{\uparrow}$ satisfies a kind of Pareto optimality given by [18, Definition 3.1]. It is shown in [10] that this condition is sufficient for existence of a risk equilibrium; see Theorem 3.1, which has a Pareto optimality framing, and the connection to both system optimization and the system risk function $v^{\mathrm{r}}$ in section 3.2 of that paper.

Statement 3, due to [15, Theorem 2.5] as already noted, is a more general version of the prior existence result established for $\mathcal{Z}=L_{\infty}(\Omega)$ in [14, Theorem 3.1].

Proof of Theorem 5. As already mentioned, we only need to prove sufficiency of statements 1 and 2. Rather than dealing with an equilibrium in the risk market, we use its characterization as a solution of the system optimization problem (6); see Theorem 1.

Assume statement 1 . In this case (6) can be written as a linear program whose feasible set is nonempty. We will show that nonemptiness of $\bigcap_{i} \operatorname{dom} r_{i}^{*}$ implies that the objective function of (6), hence of its linear programming reformulation, is bounded below on its feasible set. Since a feasible linear program that is bounded has a solution, even in infinite dimensions, the result follows.

By hypothesis, $r_{i}(Z)$, for any $Z \in \mathcal{Z}$, can be written as the minimum value of $\alpha$ such that $\alpha \geq \alpha_{j}^{i}+\zeta_{j}^{i}[Z]$ for $j=1, \ldots, J_{i}$, where $J_{i} \in \mathbb{N}$ and $\alpha_{j}^{i} \in \mathbb{R}, \zeta_{j}^{i} \in \mathcal{Z}^{* 5}$ for each $j$. Thus $r_{i}(Z)=\max \left\{\alpha_{j}^{i}+\zeta_{j}^{i}[Z]: j=1, \ldots, J_{i}\right\}$ and, for any $\zeta \in \mathcal{Z}^{*}$,

$$
r_{i}^{*}(\zeta)=\sup _{Z \in \mathcal{Z}}\left(\zeta[Z]-\max _{j=1, \ldots, J_{i}} \alpha_{j}^{i}+\zeta_{j}^{i}[Z]\right),
$$

which, by inspection, is finite valued if and only if $\sup _{Z} \zeta[Z]-\max _{j=1, \ldots, J_{i}} \zeta_{j}^{i}[Z]<\infty$. The last quantity is actually a support function $-\sigma_{\mathcal{D}_{i}-\zeta}(Z)$ where $\mathcal{D}_{i}=\operatorname{conv}\left\{\zeta_{j}^{i}\right.$ :

\footnotetext{
${ }^{5}$ Since $r_{i}$ is a risk measure, each $\zeta_{j}^{i}$ is a probability density, but we will not use that fact here.
} 
$\left.j=1, \ldots, J_{i}\right\}$ and $\mathcal{D}_{i}-\zeta$ is its translation by $-\zeta$. This is finite valued if and only if $\sigma_{\mathcal{D}_{i}-\zeta}(Z) \geq 0$, which is equivalent to $\zeta \in \mathcal{D}_{i}$. In short, dom $r_{i}^{*}=\mathcal{D}_{i}$.

By hypothesis there exists $\zeta_{0} \in \bigcap_{i=1}^{N} \operatorname{dom} r_{i}^{*}$, hence $\zeta_{0}$ lies in each $\mathcal{D}_{i}$. For any $W_{\uparrow} \in \mathcal{Z}^{N}$,

$$
\begin{aligned}
\sum_{i=1}^{N} r_{i}\left(Z_{i}-W_{i}\right) & \geq \sum_{i=1}^{N}\left(\sigma_{\mathcal{D}_{i}}\left(Z_{i}-W_{i}\right)-\max _{j=1, \ldots, J_{i}} \alpha_{j}^{i}\right) \\
& \geq\left(\sum_{i=1}^{N} \sigma_{\mathcal{D}_{i}}\left(Z_{i}-W_{i}\right)\right)-\max _{i=1, \ldots, N} \min _{j=1, \ldots, J_{i}} \alpha_{j}^{i} .
\end{aligned}
$$

Now $\sigma_{\mathcal{D}_{i}}\left(Z_{i}-W_{i}\right) \geq \zeta_{0}\left[Z_{i}-W_{i}\right]$, hence $\sum \sigma_{\mathcal{D}_{i}}\left(Z_{i}-W_{i}\right) \geq \zeta_{0}\left[\sum Z_{i}\right]$ if $W_{\uparrow}$ is feasible for (6). That is, $\sum_{i=1}^{N} r_{i}\left(Z_{i}-W_{i}\right)$ is bounded below by a scalar that is independent of $W_{\uparrow}$.

Assume statement 2. Existence of solutions of inf convolutions, sometimes called exactness, is a very well studied problem in convex analysis and we appeal here to the standard result [3]. Given a Banach space $\mathcal{X}$ and convex, lower semicontinuous mappings $f_{1}, f_{2}: \mathcal{X} \rightarrow \mathbb{R}_{\infty}$, this result says for any $x_{0} \in \mathcal{X}$ that the inf convolution $\inf _{x_{1}, x_{2} \in \mathcal{X}}\left\{f_{1}^{*}\left(x_{1}\right)+f_{2}^{*}\left(x_{2}\right): x_{1}+x_{2}=x_{0}\right\}$ is achieved as a minimum if $\operatorname{sqri}\left(\operatorname{dom} f_{1}-\operatorname{dom} f_{2}\right) \neq \emptyset$, where the latter condition follows if $\operatorname{sqri}\left(\operatorname{dom} f_{1}\right) \cap$ sqri $\left(\operatorname{dom} f_{2}\right)$ is nonempty. Without giving details of what are classical and elementary arguments, exactness of the infimal convolution over $N$ convex, lower semicontinuous mappings $f_{i}: \mathcal{X} \rightarrow \mathbb{R}_{\infty}$ follows by induction if $\bigcap_{i} \operatorname{sqri}\left(\operatorname{dom} f_{i}\right) \neq \emptyset$. This result can be applied when $\mathcal{X}=\mathcal{Z}^{*}, f_{i}=r_{i}^{*}$ for $i=1, \ldots, N$, and $f_{i}^{*}$ is the second conjugate $r_{i}^{* *}$ which is defined on $\mathcal{Z}^{* *}$. Since $r_{i}$ is continuous and convex, and $\mathcal{Z}$ is reflexive, then $\mathcal{Z}^{* *}=\mathcal{Z}$ and $r_{i}^{* *}=r_{i}$. Thus $\bigcap_{i} \operatorname{sqri}\left(\operatorname{dom} r_{i}^{*}\right) \neq \emptyset$ implies for any $Z_{0} \in \mathcal{Z}$ that $\inf _{Z_{\uparrow}}\left\{\sum_{i} r_{i}\left(Z_{i}\right): \sum_{i} Z_{i}=Z_{0}\right\}$ is achieved as a minimum. That is, there exists a solution of (7) and hence of the system risk problem (6).

An immediate corollary is for the case when all agents use CRMs $r_{i}=\sigma_{\mathcal{D}_{i}}$; hence, from Theorem $4, v^{\mathrm{r}}=\sigma_{\mathcal{D}_{0}}$ where $\mathcal{D}_{0}=\bigcap_{i} \mathcal{D}_{i}$. It is elementary that the convex conjugate of $\sigma_{\mathcal{D}_{i}}$ takes the value 0 when $\Pi \in \mathcal{D}_{i}$ and $\infty$ otherwise, that is, $\operatorname{dom} r_{i}^{*}=\mathcal{D}_{i}$ for $i=1, \ldots, N$ and $\operatorname{dom} v^{\mathrm{r} *}=\mathcal{D}_{0}$. The following result is, therefore, merely a restatement of Theorem 4 and needs no proof.

Corollary 1. Let the assumptions of Theorem 4 hold. In addition suppose each $r_{i}$ is a CRM with risk set $\mathcal{D}_{i}$ and let $\mathcal{D}_{0}=\bigcap_{i} \mathcal{D}_{i}$. Then each of the following conditions is sufficient for existence of a risk market equilibrium:

1. Each $\mathcal{D}_{i}$ is the convex hull of a finite (nonzero) number of probability densities, and $\mathcal{D}_{0} \neq \emptyset$.

2. $\mathcal{Z}$ is reflexive, e.g., either $\mathbb{R}^{K}$ or an $L_{p}$ space with $1<p<\infty$, and $\bigcap_{i}$ $\operatorname{sqri}\left(\mathcal{D}_{i}\right) \neq \emptyset$.

3. $\mathcal{Z}$ is either $\mathbb{R}^{K}$ or a nonatomic $L_{p}$ space with $1 \leq p \leq \infty$, each $r_{i}$ is law invariant, and $\mathcal{D}_{0} \neq \emptyset$.

3. Risky design equilibrium problems and risky design games. We extend the framework of a risk market to risky design equilibrium problems and then Nash games. Section 3.1 models our risky design equilibrium problem along with the risk, convexity, and technical assumptions that are needed for later results. Section 3.2 gives a chain rule, Theorem 6 , that is used to formulate the stationary conditions of design agents. Section 3.3 contains our main result, Theorem 8 , which shows how a risky design equilibrium problem, where agents use risk measures, can be reformulated 
as a risky design game given in Definition 5. In the latter, design agents act as risk neutral with respect to a probability density defined by an emergent system risk agent, which shows stochastic-endogeneity of these systems. Theorem 8 also shows existence of a risky design equilibrium under reasonable conditions. Corollary 2 specializes this to the case of CRMs.

In this section and section 4 to follow, we restrict $L_{p}$ spaces to those with $1 \leq$ $p<\infty$. The exclusion of $p=\infty$ is a limitation in the chain rule, Theorem 6 .

3.1. Risky design equilibria and modeling assumptions. Let us motivate the model for risky design equilibria in slightly more detail than the introduction. Each agent is considering building a plant today for production tomorrow. Let $x_{i} \in \mathbb{R}^{n}$ denote agent $i$ 's design variables ${ }^{6}$ for its prospective plant and $I_{i}\left(x_{i}\right)$ denote the cost of that plant. For any design $x_{i}$ the cost of operations, tomorrow, is uncertain, i.e., lies in the uncertain outcome space $\mathcal{Z}$. Those costs are also affected by other agents' investments, $x_{-i}:=\left(x_{j}\right)_{j \neq i} \in \mathbb{R}^{n(N-1)}$. Agent $i$ 's risky asset is thus denoted $\Xi_{i}\left(x_{i}, x_{-i}\right)$, where $\Xi: \mathbb{R}^{n} \times \mathbb{R}^{n(N-1)} \rightarrow \mathcal{Z}$.

Given an investment budget $b_{i} \geq 0$, agent $i$ 's strategy set is

$$
X_{i}=\left\{x_{i} \in \mathbb{R}^{n}: x_{i} \geq 0, I_{i}\left(x_{i}\right) \leq b_{i}\right\}
$$

and its strategy is determined by solving

$$
\min _{x_{i}} I_{i}\left(x_{i}\right)+r_{i}\left(\Xi_{i}\left(x_{i}, x_{-i}\right)\right) \quad \text { subject to } \quad x_{i} \in X_{i} .
$$

In fact $I_{i}$ can depend on $x_{-i}$ and also can be absorbed, by translation invariance, into $\Xi_{i}\left(x_{i}, x_{-i}\right)$ via $I_{i}\left(x_{i}\right)+r_{i}\left(\Xi_{i}\left(x_{i}, x_{-i}\right)\right)=r_{i}\left(I_{i}\left(x_{i}\right)+\Xi_{i}\left(x_{i}, x_{-i}\right)\right)$. So we will notionally simplify agent $i$ 's problem, without loss of modeling generality, to

$$
\min _{x_{i}} r_{i}\left(\Xi_{i}\left(x_{i}, x_{-i}\right)\right) \quad \text { subject to } \quad x_{i} \in X_{i}
$$

This constitutes a noncooperative Nash game, over agents $i=1, \ldots, N$, which is a game theoretic extension of risk averse optimization [31].

We follow the introduction by including risk trading and restating (2) and (4) below. Given the price of risk $P^{\mathrm{r}}$ on $\mathcal{Z}$, the $i$ th agent finds $x_{i} \in \mathbb{R}^{n}$ and $W_{i} \in \mathcal{Z}$ to solve

$$
\min _{x_{i}, W_{i}} P^{\mathrm{r}}\left[W_{i}\right]+r_{i}\left(\Xi_{i}\left(x_{i}, x_{-i}\right)-W_{i}\right) \quad \text { subject to } \quad x_{i} \in X_{i} .
$$

Also, risk trades must be balanced:

$$
\sum_{i=1}^{N} W_{i}=0 .
$$

Applying Definition 1, the system (11) (for $i=1, \ldots, N$ ) and (12) is a risky design equilibrium problem.

Our goal is to extend the existence theory for Nash games under risk to the new paradigm of risky design equilibrium problems. Our assumptions follow.

\footnotetext{
${ }^{6}$ To simplify notation we assume $n$ does not depend on $i$. Our results hold when each $x_{i}$ has a possibly different dimension $n_{i}$ by appropriate substitution of $n_{i}$ for $n$ or embedding $\mathbb{R}^{n_{i}}$ in $\mathbb{R}^{n}$, where $n=\max _{i} n_{i}$.
} 


\section{Risk assumptions.}

1. By $\mathcal{Z}$ we denote an uncertain outcome space, i.e., either $\mathbb{R}^{K}$ or $L_{p}(\Omega)$ for $p \in[1, \infty)$, where $(\Omega, \mathcal{F}, \mu)$ is nonnegative, nontrivial, finite measure space.

2. We study a risk market of $N$ agents where each agent $i$ assesses the cost of uncertain outcomes by a (convex) risk measure $r_{i}: \mathcal{Z} \rightarrow \mathbb{R}$ (section 2.2, Definition 3).

3. The market for risk is perfectly competitive and, except for section 4 , complete (section 2.1.2).

Convexity assumptions.

1. For each $i=1, \ldots, N$ let $X_{i}$ be a nonempty compact convex set in $\mathbb{R}^{n}$,

$$
X_{\uparrow}:=X_{1} \times \ldots \times X_{N} \subset \mathbb{R}^{n N} \text {. }
$$

2. Given $x_{\uparrow} \in X_{\uparrow}, \Xi_{i \omega}\left(\cdot, x_{-i}\right)$ is real valued and convex on $X_{i}$ for each $i$ and $\omega$. Technical assumptions.

1. Each $\Xi_{i}: X_{\uparrow} \rightarrow \mathcal{Z}$ is continuous.

2. Assume further that, for any $x_{\uparrow} \in X_{\uparrow}, \Xi_{i}\left(\cdot, x_{-i}\right)$ is directionally differentiable at $x_{i}$ in any direction $x$ in the relative interior of $T_{X_{i}}\left(x_{i}\right), \operatorname{rint} T_{X_{i}}\left(x_{i}\right)$.

Note that these assumptions are sufficient for existence of a solution of the Nash game (10), e.g., convexity of $r_{i}\left(\Xi_{i}\left(\cdot, x_{-i}\right)\right)$ follows from convexity of each $\Xi_{i \omega}\left(\cdot, x_{-i}\right)$ given monotonicity of $r_{i}$ [31] , and $r_{i}\left(\Xi_{i}\left(x_{i}, x_{-i}\right)\right)$ is obviously continuous in $\left(x_{i}, x_{-i}\right)$.

For clarity we state Nash's theorem as Theorem 14 in the appendix, where, in keeping with the original statement [24], agent $i$ 's objective function is required to be quasi-convex in its strategy $x_{i}$. In this paper, we unavoidably require convexity in $x_{i}$ in order to use equivalence between stationarity and global optimality.

3.2. Chain rule. Here we provide tools to derive the stationary conditions of each agent's optimization problem. Hence we consider a single agent only, omit the index $i$, and modify and extend results developed for optimization under risk in [31, 33].

Let $\rho$ denote the composite function $r \circ \Xi(x):=r(\Xi(x))$, where $r: \mathcal{Z} \rightarrow \mathbb{R}$ is a risk measure, each $\Xi_{\omega}: \mathbb{R}^{n} \rightarrow \mathbb{R}_{\infty}$ is lower semicontinuous and convex with the same domain $D$, and $\rho$ takes the value $\infty$ outside $D$. If we are minimizing $\rho$, then we would like to formulate stationary conditions using gradients or subgradients of $\rho$ via a chain rule, Theorem 6 below, which will be used in section 3.3. To clarify what is needed, we mention a result which follows from classical finite dimensional convex analysis [29]. If $\mathcal{Z}=\mathbb{R}^{K}, \bar{x} \in D, \bar{Z}=\Xi(\bar{x})$, and each $\Xi_{\omega}$ is subdifferentiable at $\bar{x}$, then we have the chain rule

$$
\partial \rho(\bar{x})=\{\zeta[\xi]: \zeta \in \partial r(\bar{Z}), \xi \in \partial \Xi(\bar{x})\},
$$

where $\partial \Xi(\bar{x}):=\partial \Xi_{1}(\bar{x}) \times \ldots \times \partial \Xi_{K}(\bar{x})$. The fact that $\partial r(\bar{Z})$ consists of probability densities allows us to rewrite this as $\partial \rho(\bar{x})=\left\{\mathbb{E}_{\Pi}[\xi]: \Pi \in \partial r(\bar{Z}), \xi \in \partial \Xi(\bar{x})\right\}$. We need to extend this chain rule by allowing, first, risk trading, i.e., composite functions which map $(x, W) \in \mathbb{R}^{n} \times \mathcal{Z}$ to $r(\Xi(x)-W)$, and, second, infinite dimensional uncertainties, $\mathcal{Z}=L_{p}(\Omega)$.

We give a chain rule that combines and extends two results given in [31], namely, a chain rule Theorem 6.11 and disintegration result Theorem 7.47. We depart from the former by studying compositions that may take the value $\infty$ and by showing closedness of the set on the right-hand side of the subdifferential formula (14), below.

THeOREM 6. Let $\mathcal{Z}$ be an uncertain outcome space and $r: \mathcal{Z} \rightarrow \mathbb{R}$ be a (continuous) risk measure. Let $D$ be a nonempty closed convex set in $\mathbb{R}^{n}$ and, for each 
$\omega$, let $\Xi_{\omega}: \mathbb{R}^{n} \rightarrow \mathbb{R}_{\infty}$ be a convex and lower semicontinuous function with domain $D$ such that $\Xi(x):=\left(\Xi_{\omega}(x)\right)_{\omega}$ defines a continuous mapping from $D$ to $\mathcal{Z}$. Define the composite function $\rho: \mathbb{R}^{n} \times \mathcal{Z} \rightarrow \mathbb{R}_{\infty}$ by

$$
\rho(x, W):= \begin{cases}r(\Xi(x)-W) & \text { for } x \in D \text { and } W \in \mathcal{Z}, \\ \infty & \text { otherwise. }\end{cases}
$$

1. The composition $\rho$ is convex and lower semicontinuous and its domain is $D \times \mathcal{Z}$.

2. Suppose in addition that $\bar{x} \in D$ is such that there exists the directional derivative $\Xi^{\prime}(\bar{x} ; x) \in \mathcal{Z}$ for each $x \in \operatorname{rint} T_{D}(\bar{x})$. Then for any $\bar{W} \in \mathcal{Z}$ and $\bar{Z}=\Xi(\bar{x})-\bar{W}$,

(a) if $\mathcal{Z}=\mathbb{R}^{K}$ and $\partial \Xi(\bar{x}):=\partial \Xi_{1}(\bar{x}) \times \cdots \times \partial \Xi_{K}(\bar{x})$ we have

$$
\partial \rho(\bar{x}, \bar{W})=\left\{\left(\mathbb{E}_{\Pi}[\xi],-\Pi\right): \xi \in \partial \Xi(\bar{x}), \Pi \in \partial r(\bar{Z})\right\} ;
$$

(b) if $\mathcal{Z}=L_{p}(\Omega, \mathcal{F}, \mu)$, where $1 \leq p<\infty$, we have

$$
\partial \rho(\bar{x}, \bar{W})=\bigcup_{\Pi \in \partial r(\bar{Z})}\left(\mathbb{E}_{\Pi}[\partial \Xi(\bar{x})] \times\{-\Pi\}\right),
$$

where

$$
\mathbb{E}_{\Pi}[\partial \Xi(\bar{x})]:=\left\{\mathbb{E}_{\Pi}[\xi]: \xi_{\omega} \in \partial \Xi_{\omega}(\bar{x}) \text { for all } \omega,\left(\Pi_{\omega} \xi_{\omega}\right)_{\omega} \text { is integrable }\right\} .
$$

Due to the highly technical nature of the proof, we postpone it to the appendix.

Note that, in the situation of Theorem 6, a sufficient condition for the directional differentiability property of $\Xi$ required in part 2 is that $\bar{x}$ lies in $\operatorname{rint} D[33$, Theorem 7.44].

3.3. Reformulation of a risky design equilibrium problem as a Nash game.

3.3.1. A link to risk neutral Nash games via stochastic-endogeneity. Recall the system risk function $v^{\mathrm{r}}$ (see (7) in section 2.1.2), which is a risk measure (Theorem 4 in section 2.2.1).

TheOREm 7. Suppose the risk, convexity, and technical assumptions hold. Consider the following two statements:

A. $\left(x_{\uparrow} W_{\uparrow}, \Pi\right)$ is a risky design equilibrium, i.e., solves $(11),(12)$, where $P^{\mathrm{r}}=\Pi$.

B. $\left(x_{\uparrow}, \Pi\right)$ solves the equilibrium system (16), (17):

$$
\min _{x_{i}} \mathbb{E}_{\Pi}\left[\Xi_{i}\left(x_{i}, x_{-i}\right)\right] \quad \text { subject to } \quad x_{i} \in X_{i}
$$

and

$$
\Pi \in \partial v^{\mathrm{r}}\left(\sum_{i=1}^{N} \Xi_{i}\left(x_{i}, x_{-i}\right)\right) .
$$

Then $A \Rightarrow B$, and the converse holds (for appropriate $W_{\uparrow}$ ) if the risk market has an equilibrium when each agent $i$ holds the asset $Z_{i}=\Xi_{i}\left(x_{i}, x_{-i}\right)$.

Copyright $@$ by SIAM. Unauthorized reproduction of this article is prohibited. 
Theorem 7 stands without boundedness of $X_{i}$ (i.e., replace compact by closed) in Assumption 5.

Remark 1. The reformulation in statement $\mathrm{B}$ allows us to ignore risk trades. The design agents $(i=1, \ldots, N)$ are standard risk neutral optimizers, $(16)$, and there is a final equilibrium condition, (17), that determines the risk neutral probability density. Hence this format is a stochastic-endogenous equilibrium problem. We contrast this with the more traditional stochastic game in which (17) is dropped in favor of a fixed probability density $\Pi$, given in advance, that results in a risk neutral Nash game (16).

Proof of Theorem 7. Extend $\Xi_{i \omega}\left(x_{i}, x_{-i}\right)$ to take the value $+\infty$ for $x_{\uparrow} \notin X_{\uparrow}$, and define $\rho_{i}\left(x_{i}, W_{i}\right)=r_{i}\left(\Xi_{i}\left(x_{i}, x_{-i}\right)-W_{i}\right)$ for $\left(x_{i}, W_{i}\right) \in X_{i} \times \mathcal{Z}$ and $\infty$ otherwise. Then the stationary condition of (11) can be formulated as $0 \in\left(0, P^{\mathrm{r}}\right)+\partial \rho_{i}\left(x_{i}, W_{i}\right)$, which, by the chain rule Theorem 6 , gives, ${ }^{7}$ for some $\Pi_{i} \in \partial r_{i}\left(\Xi_{i}\left(x_{i}, x_{-i}\right)-W_{i}\right)$,

$$
\begin{aligned}
& 0 \in \mathbb{E}_{\Pi_{i}}\left[\partial_{x_{i}} \Xi_{i}\left(x_{i}, x_{-i}\right)\right], \\
& 0=P^{\mathrm{r}}-\Pi_{i} .
\end{aligned}
$$

This shows that $P^{\mathrm{r}}$ is independent of $i$, and we simplify the conditions to

$$
\begin{aligned}
& 0 \in \mathbb{E}_{\Pi}\left[\partial_{x_{i}} \Xi_{i}\left(x_{i}, x_{-i}\right)\right], \\
& \Pi \in \partial r_{i}\left(\Xi_{i}\left(x_{i}, x_{-i}\right)-W_{i}\right) .
\end{aligned}
$$

Suppose $\left(x_{\uparrow}, W_{\uparrow}, \Pi\right)$ is a risky design Nash equilibrium so that (18) holds with $P^{\mathrm{r}}=\Pi$. Obviously $W_{\uparrow}$ and $\Pi$ form a risk equilibrium for the risky assets $Z_{i}=$ $\Xi_{i}\left(x_{i}, x_{-i}\right), i=1, \ldots, N$, and hence Theorem 2 gives (17). Also, the first inclusion of (18) is stationarity of (16) by the chain rule Theorem 6 , i.e., $x_{i}$ solves this convex problem.

Conversely take $x_{\uparrow}, \Pi$ that solve (16), (17). We have seen that the stationary condition of (16) is the first inclusion of (18). Assuming further that there exists a risk equilibrium given $Z_{i}=\Xi_{i}\left(x_{i}, x_{-i}\right)$ for each $i$, Theorem 2 ensures that $\Pi$ prices risk for $Z_{\uparrow}$. Let $W_{\uparrow}$ be the equilibrium risk trades. Hence, by Theorem 1 , the second inclusion of (18) holds for each $i$ when $P^{\mathrm{r}}=\Pi$. Thus we have solved (11). Since $W_{\uparrow}$ must also be balanced we are done.

3.3.2. A risky design equilibrium problem is equivalent to a Nash game. Recall the convex conjugate of $v^{\mathrm{r}}$, the function $v^{\mathrm{r} *}: \mathcal{Z}^{*} \rightarrow \mathbb{R} \cup\{\infty\}$ defined by

$$
v^{\mathrm{r} *}(\zeta):=\sup _{Z} \zeta[Z]-v^{\mathrm{r}}(Z)
$$

A standard result of convex conjugacy for lower semicontinuous convex functions like $v^{\mathrm{r}}$ is that $\zeta \in \partial v^{\mathrm{r}}(Z)$ if and only if $Z \in \partial v^{\mathrm{r} *}(\zeta)$; see [29, Corollary 12A]. Thus the inclusion (17) is equivalent to $\sum_{i} \Xi_{i}\left(x_{i}^{k}, x_{-i}^{k}\right)$ being a member of $\partial v^{\mathrm{r} *}(\Pi)$, which, in turn, is the stationary condition for

$$
\min _{\Pi} v^{\mathrm{r} *}(\Pi)-\Pi\left[\sum_{i} \Xi_{i}\left(x_{i}^{k}, x_{-i}^{k}\right)\right] .
$$

\footnotetext{
${ }^{7}$ The first inclusion could be written $0 \in \mathbb{E}_{\Pi}\left[\partial_{x_{i}} \Xi_{i}\left(x_{i}, x_{-i}\right)\right]+N_{X_{i}}\left(x_{i}\right)$, where $N_{X_{i}}\left(x_{i}\right)$ is the normal cone of $X_{i}$ at $x_{i}$. However, adding $N_{X_{i}}\left(x_{i}\right)$ is superfluous because, due to the extended real valued definition of each $\Xi_{i \omega}$ and hence of $\mathbb{E}_{\Pi}\left[\Xi_{i}\left(x_{i}, x_{-i}\right)\right]$, the subdifferential $\mathbb{E}_{\Pi}\left[\partial_{x_{i}} \Xi_{i}\left(x_{i}, x_{-i}\right)\right]$ contains $N_{X_{i}}\left(x_{i}\right)$.
} 
There is no generality lost in restricting the decision set of this problem to the domain of $v^{\mathrm{r} *}$. Likewise, since any stationary point $\Pi$ is a subgradient of the risk measure $v^{\mathrm{r}}$, it is a probability density. Hence if $\mathcal{D}^{\mathrm{r}}$ denotes domain of $v^{\mathrm{r} *}$,

$$
\mathcal{D}^{\mathrm{r}}=\left\{\Pi \in \mathcal{P}: v^{\mathrm{r} *}(\Pi)<\infty\right\},
$$

we consider

$$
\min _{\Pi} v^{\mathrm{r} *}(\Pi)-\mathbb{E}_{\Pi}\left[\sum_{i} \Xi_{i}\left(x_{i}^{k}, x_{-i}^{k}\right)\right] \quad \text { subject to } \quad \Pi \in \mathcal{D}^{\mathrm{r}},
$$

which is equivalent to (17) but has the form required for an agent in a Nash game. In fact (19) can be viewed as an $N+1$ st agent that prices risk, the so-called system risk agent.

We summarize as follows: The risky design equilibrium problem (11) (for $i=$ $1, \ldots, N)$ and (12) in statement B of Theorem 7 can be equivalently written as the Nash game that combines (16) (for $i=1, \ldots, N$ ) and (19). This gives part 1 of Theorem 8 to follow, which, as a corollary of Theorem 7 , needs no proof.

Definition 5. The Nash game (16), with design decisions $x_{i}$ for $i=1, \ldots, N$, and (19), with a risk pricing decision $\Pi$, is called a risky design game.

We say that the risk market always has an equilibrium to mean that risk can be priced for any stream of assets $Z_{\uparrow} \in \mathcal{Z}^{N}$.

THEOREM 8. Let the risk, convexity, and technical assumptions hold and $\mathcal{D}^{\mathrm{r}}$ be the domain of $v^{\mathrm{r} *}$.

1. Consider the following statements:

A. $\left(x_{\uparrow}, W_{\uparrow}, \Pi\right)$ is a risky design equilibrium, i.e., solves (11), (12).

B. $\left(x_{\uparrow}, \Pi\right)$ solves the risky design game (16), (19).

Then $\mathrm{A} \Longrightarrow \mathrm{B}$ and the converse holds, for some $W_{\uparrow}$, if the risk market has an equilibrium when each agent $i$ holds the asset $Z_{i}=\Xi_{i}\left(x_{i}, x_{-i}\right)$.

2. If $\mathcal{D}^{\mathrm{r}}$ is closed, then statement $B$ holds: there exists a Nash equilibrium $\left(x_{\uparrow}, \Pi\right)$.

Part 1 is already proved above. In finite dimensions, existence in part 2 is evidently a direct application of Nash's theorem because $\mathcal{D}^{\mathrm{r}}$ inherits compactness from its superset $\mathcal{P}$. A complicating factor when $\mathcal{Z}=L_{p}(\Omega)$ is that the dual elements lie in $\mathcal{Z}^{*}=L_{q}(\Omega)$ and, consequently, we lack norm compactness of the closed convex set $\mathcal{P}$. This is overcome by using the weak ${ }^{*}$ topology on $\mathcal{Z}^{*}$, under which $\mathcal{P}$ is weak* compact.

Proof of Theorem 8. As noted above, we only need to prove part 2. This is an application of Nash's theorem, stated as theorem 14 in the appendix, to the game with $N+1$ agents given by (16) for $i=1, \ldots, N$, and (19) for $i=N+1$. As well as the norm, or strong, topology on $\mathcal{Z}^{*}$ we need to consider the weak topology on $\mathcal{Z}^{*}$. We need the standard result (Alaoglu's theorem, or see [28, Chapter III.7, Theorem 6]) that weak* compactness holds for every set in $\mathcal{Z}^{*}$ that is closed and bounded with respect to the usual (dual) norm on $\mathcal{Z}^{*}$. By the product topology we mean the topology on $\mathbb{R}^{n N} \times \mathcal{Z}^{*}$ whose neighborhoods are Cartesian products of neighborhoods in $\mathbb{R}^{n}$ and weak* neighborhoods in $\mathcal{Z}^{*}$.

We list the immediate properties of the game: Each agent $i=1, \ldots, N$ has a strategy set $X_{i}$ that is nonempty convex and compact (in $\mathbb{R}^{n}$ ) and an objective $\mathbb{E}_{\Pi}\left[\Xi_{i}\left(x_{i}, x_{-i}\right)\right]$ that is continuous and convex in $x_{i}$ given $\left(x_{i}, \Pi\right)$ and is also continuous in all variables $\left(x_{i}, x_{-i}, \Pi\right)$ with respect to the product topology. The last statement 
relies on continuity of $\Xi_{i}$ as a mapping from the normed space $\mathbb{R}^{n N}$ to the normed space $\mathcal{Z}$. Agent $N+1$ has as its strategy set the domain $\mathcal{D}^{\mathrm{r}}$ of the conjugate $v^{\mathrm{r} *}$ of the system risk function, and $\mathcal{D}^{\mathrm{r}}$ is convex and closed (by hypothesis) but not weak* compact because it is not in general bounded; we'll return to this later. Its objective function $\Phi\left(\Pi, x_{\uparrow}\right):=v^{\mathrm{r} *}(\Pi)-\mathbb{E}_{\Pi}\left[v^{\mathrm{r}}\left(\sum_{i=1}^{N} \Xi_{i}\left(x_{i}, x_{-i}\right)\right)\right]$ is the sum of a convex function and a linear function of $\Pi$ and hence is convex in $\Pi$. To show that $\Phi$ is lower semicontinuous in $\left(\Pi, x_{\uparrow}\right)$, we start by observing that the first term $v^{\mathrm{r} *}$, being a convex conjugate, is automatically weak* lower semicontinuous on $\mathcal{Z}^{*} .{ }^{8}$ The second (expectation) term is actually continuous in the product topology due to continuity of $v^{\mathrm{r}}$ and norm continuity of each $\Xi_{i}$. It follows that $\Phi$ is both lower semicontinuous (in the product topology) and continuous in $x_{\uparrow}$ only.

To apply Nash's theorem it is only left to show that the risk pricing problem (19) can be written as optimization over a convex weak* compact set. Recall from previous discussion that $\Pi$ lies in $\partial v^{\mathrm{r}}\left(\sum_{i=1}^{N} \Xi_{i}\left(x_{i}, x_{-i}\right)\right)$ if and only if it solves (19); and of course, such a vector $\Pi$ lies in $\mathcal{D}^{\mathrm{r}}$. Since $v^{\mathrm{r}}$ is continuous and convex, $\partial v^{\mathrm{r}}(Z)$ is uniformly bounded with respect to $Z$ near any $Z_{0} \in \mathcal{Z}$. Next, continuity of $\sum_{i=1}^{N} \Xi_{i}$ yields (norm) compactness of the image $\left\{\sum_{i=1}^{N} \Xi_{i}\left(x_{i}, x_{-i}\right): x_{\uparrow} \in X_{\uparrow}\right\}$ of the compact set $X_{\uparrow}$. Combining these two facts gives norm boundedness of the set $\cup_{x_{\uparrow} \in X_{\uparrow}} \partial v^{\mathrm{r}}\left(\sum_{i=1}^{N} \Xi_{i}\left(x_{i}, x_{-i}\right)\right)$. Let $\mathcal{D}$ be the closed convex hull of this set, which is a weak* compact subset of $\mathcal{D}^{\mathrm{r}}$ since the latter is closed by hypothesis. Thus for any $x_{\uparrow} \in X_{\uparrow}$, the strategy set $\mathcal{D}^{\mathrm{r}}$ of (19) can replaced by $\mathcal{D}$ without changing the set of optimal solutions.

An immediate corollary is for the case when all agents use CRMs $r_{i}=\sigma_{\mathcal{D}_{i}}$ and hence from Theorem $4, v^{\mathrm{r}}=\sigma_{\mathcal{D}_{0}}$, where $\mathcal{D}_{0}=\bigcap_{i} \mathcal{D}_{i}$. It is elementary that the convex conjugate of $\sigma_{\mathcal{D}_{0}}$ takes the value 0 when $\Pi \in \mathcal{D}_{0}$ and $\infty$ otherwise, hence $\mathcal{D}^{\mathrm{r}}=\mathcal{D}_{0}$, which is a closed set, and the system risk agent's problem (19) reduces to

$$
\max _{\Pi} \mathbb{E}_{\Pi}\left[\sum_{i=1}^{N} \Xi_{i}\left(x_{i}, x_{-i}\right)\right] \quad \text { subject to } \quad \Pi \in \mathcal{D}_{0} .
$$

Both parts of the next result also rely on sufficient conditions for existence of risk equilibria given by Corollary 1 in section 2.3.

COROLLARY 2. In the situation of Theorem 8 , suppose that all agents use CRMs as in Definition 4 and the system risk set $\mathcal{D}_{0}=\bigcap_{i=1}^{N} \mathcal{D}_{i}$ is nonempty. If either each risk set $\mathcal{D}_{i}$ is polyhedral and convex or the system risk set has interior relative to $\mathcal{P}$, then

1. the risky design equilibrium problem (11), (12) is equivalent to the risky design game (16), (20);

2. an equilibrium exists for each of these systems.

Remark 2. A key point regarding existence of equilibria is that we are merely exercising long established ideas and that standard extensions or elaborations are equally possible. One extension asks when boundedness of the strategy sets $X_{i}$ can be dispensed with. If we can show that equilibria, should they exist, lie in a bounded set, then the original proof holds trivially. (This idea is exemplified in the proof of Theorem 8, part 2 in the appendix.) A more subtle approach imposes conditions on the variational inequality that is formed by writing all agents' stationary conditions in

\footnotetext{
${ }^{8}$ Let $f: \mathcal{Z} \rightarrow \mathbb{R}_{\infty}$ and look at the epigraph of $f^{*},\left\{(\zeta, \alpha) \in \mathcal{Z}^{*} \times \mathbb{R}: f^{*}(\zeta) \leq \alpha\right\}$. This can be written as the intersection, over all $Z \in \mathcal{Z}$, of the weak* closed sets (half spaces) $\left\{(\zeta, \alpha) \in \mathcal{Z}^{*} \times \mathbb{R}\right.$ : $\zeta[Z]-f(Z) \leq \alpha\}$.
} 
one system; see [13, Chapter 2] or [17, section 7]. The direct analysis of the equilibrium conditions may prove challenging since the associated variational inequality problem has expectation-valued maps. Consequently, sufficiency conditions for existence are by no means easy to ascertain, though some recent work [27] may assist with respect to coercivity. Sufficiency conditions are a topic for further work.

4. Incomplete risky design equilibrium problems. Our final section takes an introductory look at risky design problems when the risk market is incomplete. Suppose risk trades are confined to a closed subspace $\mathcal{W}$ of $\mathcal{Z}$, which is natural in financial markets where there are several kinds of financial securities that define the basis of $\mathcal{W}$. This is the case in [22], which, however, confines itself to financial trades to study hedging.

Adapting from the complete case (2) or (11), given $x_{-i}$ and $P^{\mathrm{r}}$, agent $i$ solves

$$
\min _{x_{i}, W_{i}} P^{\mathrm{r}}\left[W_{i}\right]+r_{i}\left(\Xi_{i}\left(x_{i}, x_{-i}\right)-W_{i}\right) \quad \text { subject to } \quad x_{i} \in X_{i}, \quad W_{i} \in \mathcal{W} .
$$

The price of risk $P^{\mathrm{r}}$ is a dual element in $\mathcal{W}^{*}$ (since it only acts on $\mathcal{W}$ ).

DeFinition 6. The incomplete risky design equilibrium problem is the system that combines (21) (for $i=1, \ldots, N$ ) with balanced risk trades, $\sum_{i} W_{i}=0 . \quad A n$ incomplete risky design equilibrium is a solution of this system that comprises design variables $x_{\uparrow} \in X_{\uparrow}$, risk trades $W_{\uparrow} \in \mathcal{W}^{N}$ and a price of risk $P^{\mathrm{r}} \in \mathcal{W}^{*}$.

The main results are Theorem 9 , which is a straightforward extension of classical results such as Theorem 1 to incomplete risk markets (cf. [18]); Theorem 10, which extends Corollary 1 on existence of risk equilibria when agents use CRMs to the incomplete case (see, for example, [15]); Theorem 11, which shows - analogous to Theorem 7-how an incomplete risky design equilibrium problem can be reformulated as a combination of a risk neutral design game and a risk pricing process; and Theorem 12 - analogous to Corollary 2-which gives sufficient conditions for existence of an incomplete risky design equilibrium when there are only finitely many stochastic scenarios and agents use CRMs.

Theorems 11 and 12 show the stochastic-endogenous nature of incomplete risky design equilibria but differ fundamentally from the case of complete markets in that each design agent may select a different probability density or marginal price of risk (see Definition 7).

4.1. Review of incomplete risk markets. Suppose there are $N$ agents with risky assets $Z_{1}, \ldots, Z_{N} \in \mathcal{Z}$. In the risk market, given $P^{\mathrm{r}}$, agent $i$ faces the problem

$$
\min _{W_{i}} P^{\mathrm{r}}\left[W_{i}\right]+r_{i}\left(Z_{i}-W_{i}\right) \quad \text { subject to } \quad W_{i} \in \mathcal{W} .
$$

As above, $P^{\mathrm{r}} \in \mathcal{W}^{*}$ is such that, at equilibrium, $W_{\uparrow}$ is balanced.

We give a straightforward extension of Theorem 2. Denote by $\Pi_{\uparrow}$ the list of probability densities $\left(\Pi_{i}\right)_{i=1}^{N}$. Let $\left.\zeta\right|_{\mathcal{W}}$ denote the restriction of a dual element $\zeta \in \mathcal{Z}^{*}$ to $\mathcal{W}$, so that $\left.\zeta\right|_{\mathcal{W}} \in \mathcal{W}^{*}$. Likewise for a set $S \subset \mathcal{Z}^{*}$, let $\left.S\right|_{\mathcal{W}}:=\left\{\left.\zeta\right|_{\mathcal{W}}: \zeta \in S\right\}$.

Theorem 9. Suppose the risk, convexity, and technical assumptions hold, and $\mathcal{W}$ is a nonempty closed subspace of $\mathcal{Z}$. If an incomplete risk market equilibrium exists for a given list $Z_{\uparrow} \in \mathcal{Z}^{N}$ of agents' endowments, then the following are equivalent:

1. $P^{\mathrm{r}}$ prices risk for the incomplete market.

2. $W_{\uparrow}$ solves the system risk problem, 


$$
\min _{W_{\uparrow}} \sum_{i=1}^{N} r_{i}\left(Z_{i}-W_{i}\right) \quad \text { subject to } \quad W_{\uparrow} \in \mathcal{W}^{N}, \quad \sum_{i=1}^{N} W_{i}=0,
$$

and $P^{\mathrm{r}}$ is the Lagrange multiplier of the trade balance constraint.

3. $W_{\uparrow}$ is balanced and $P^{\mathrm{r}}$ is such that

$$
P^{\mathrm{r}} \in \bigcap_{i=1}^{N}\left(\left.\partial r_{i}\left(Z_{i}-W_{i}\right)\right|_{\mathcal{W}}\right) .
$$

A proof isn't needed as it is more or less the same as in the complete case: As in Theorem 1, the equivalence between statements 1 and 2 is classical economics, and the equivalence between all statements can be directly derived by looking at the stationary conditions of the convex optimization problems that are involved.

When investigating existence later we will assume agent risk preferences are represented by CRMs. The next result is a direct extension of Corollary 1 to the incomplete case. The proofs of parts 1 and 2 replicate the proofs of their counterparts for Theorem 7 by merely adding the constraint $W_{\uparrow} \in \mathcal{W}^{N}$. The proof of part 3 is an adaptation of $[15$, section 7$]$.

THEOREM 10. Let the assumptions of Theorem 9 hold. In addition suppose for each $i$ that $r_{i}$ is a CRM with closed convex risk set $\mathcal{D}_{i}$. Then each of the following conditions is sufficient for existence of a risk market equilibrium:

1. Each $\mathcal{D}_{i}$ is the convex hull of a finite (nonzero) number of probability densities, and $\bigcap_{i} \mathcal{D}_{i}$ is nonempty.

2. $\mathcal{Z}$ is reflexive, e.g., either $\mathbb{R}^{K}$ or an $L_{p}$ space with $1<p<\infty$, and $\bigcap_{i} \operatorname{sqri}\left(\mathcal{D}_{i}\right)$ is nonempty.

3. $\mathcal{Z}$ is either $\mathbb{R}^{K}$ or a nonatomic $L_{p}$ space with $1 \leq p \leq \infty$, each $r_{i}$ is law invariant, and $\bigcap_{i} \mathcal{D}_{i}$ is nonempty.

4.2. Reformulation and existence of solutions of incomplete risky design equilibrium problems. Recall the risk neutral design problem (1) or (16) that we adapt here to account for different probability densities $\Pi_{i}$ :

$$
\min _{x_{i}} \mathbb{E}_{\Pi_{i}}\left[\Xi_{i}\left(x_{i}, x_{-i}\right)\right] \quad \text { subject to } \quad x_{i} \in X_{i} .
$$

We couple this with a risk market characterization derived from statement 3 of Theorem 9 .

Definition 7. Given risky assets $Z_{\uparrow} \in \mathcal{Z}^{N}$, we say $\Pi_{\uparrow} \in \mathcal{W}^{*}$ is a marginal risk equilibrium for $Z_{\uparrow}$ if there exist a balanced list $W_{\uparrow} \in \mathcal{Z}^{N}$ and $P^{\mathrm{r}} \in \mathcal{W}^{*}$ such that, for all $i$,

$$
\begin{aligned}
& \Pi_{i} \in \partial r_{i}\left(Z_{i}-W_{i}\right), \\
& P^{\mathrm{r}}=\Pi_{i} \mid \mathcal{W} .
\end{aligned}
$$

THEOREM 11. In the setting of Theorem 9 , let $\Xi_{\uparrow}\left(x_{\uparrow}\right):=\left(\Xi_{i}\left(x_{i}, x_{-i}\right)\right)_{i=1}^{N}$ for $x_{\uparrow} \in X_{\uparrow}$. Then $\left(x_{\uparrow}, W_{\uparrow}, P^{\mathrm{r}}\right)$ is an incomplete risky design equilibrium if and only if $x_{\uparrow}$ solves the risk neutral Nash design equilibrium (23), where $\Pi_{\uparrow}$ is a marginal risk equilibrium for $Z_{\uparrow}=\Xi_{\uparrow}\left(x_{\uparrow}\right)$ and $P^{\mathrm{r}}=\left.\Pi_{i}\right|_{\mathcal{W}}$ for any $i$.

Proof. Following the proof of Theorem 7 we extend $\Xi_{i \omega}\left(x_{i}, x_{-i}\right)$ to take the value $+\infty$ for $x_{\uparrow} \notin X_{\uparrow}$, define $\rho_{i}\left(x_{i}, W_{i}\right)=r_{i}\left(\Xi_{i}\left(x_{i}, x_{-i}\right)-W_{i}\right)$ for $\left(x_{i}, W_{i}\right) \in X_{i} \times \mathcal{Z}$ 
and $\infty$ otherwise, and use the chain rule Theorem 6 to give the stationary condition of (21) as the existence of $\Pi_{i} \in \partial r_{i}\left(\Xi_{i}\left(x_{i}, x_{-i}\right)-W_{i}\right)$ such that

$$
\begin{aligned}
& 0 \in \mathbb{E}_{\Pi_{i}}\left[\partial_{x_{i}} \Xi_{i}\left(x_{i}, x_{-i}\right)\right], \\
& 0=P^{\mathrm{r}}-\Pi_{i} \mid \mathcal{W} .
\end{aligned}
$$

Thus if $\left(x_{\uparrow}, W_{\uparrow}, P^{\mathrm{r}}\right)$ is an incomplete risky design equilibrium then $x_{i}$ solves (23) for each $i,(24)$ holds, and $\sum_{i} W_{i}=0$. The converse follows with the help of Theorem 9.

Theorem 11 puts the incomplete risky design equilibrium problem into a form suitable for application of Kakutani's fixed point theorem, stated as Theorem 13 in the appendix. This leads to our final existence result, which is limited to finite dimensions.

As in [22], we need an interiority condition on $\mathcal{D}_{0}=\bigcap_{i} \mathcal{D}_{i}$. Given $\mathcal{D} \subset \mathcal{P}$ we write $\operatorname{int}_{\mathcal{P}}(\mathcal{D})$ for the interior of $\mathcal{D}$ relative to $\mathcal{P}$, which the set of $\Pi \in \mathcal{D}$ such that $\mathcal{D}$ contains a neighborhood of $\Pi$ intersected with $\mathcal{P}$. Nonemptiness of $\operatorname{int}_{\mathcal{P}}\left(\mathcal{D}_{0}\right)$ ensures boundedness of risk trades; the analogous condition [22, Assumption B] plays the same role in that paper.

THEOREM 12. In the setting of Theorem 9 , suppose $\mathcal{Z}=\mathbb{R}^{K}$; each $r_{i}$ is a CRM with a closed convex risk set $\mathcal{D}_{i}$; and $\operatorname{int}_{\mathcal{P}}\left(\mathcal{D}_{0}\right) \neq \emptyset$. Then there exists an incomplete risky design equilibrium.

Proof. For each $i=1, \ldots, N$, given $x_{-i} \in X_{-i}$ and $\Pi_{i} \in \mathcal{D}_{i}$, let $\Phi_{i}\left(x_{-i}, \Pi_{i}\right)$ be the set of optimal solutions $x_{i}$ of (23) which is nonempty, compact, and convex.

Given $x_{\uparrow} \in X_{\uparrow}$, consider the risk market corresponding to risky assets $Z_{i}=$ $\Xi_{i}\left(x_{i}, x_{-i}\right)$ for $i=1, \ldots, N$. Since $\operatorname{sqri}\left(\mathcal{D}_{i}\right)$ contains $\operatorname{int}_{\mathcal{P}}\left(\mathcal{D}_{i}\right)$ for each $i$, we appeal to statement 2 of Theorem 10 to get a risk equilibrium corresponding to $Z_{\uparrow}$. Hence there exists a marginal risk equilibrium $\Pi_{\uparrow}$ for $Z_{\uparrow}$ from statement 3 of Theorem 9 . Let $\Phi_{N+1}\left(x_{\uparrow}\right)$ be the set of such $\Pi_{\uparrow}$. Then $\Phi_{N+1}\left(x_{\uparrow}\right)$ is a nonempty, convex, and compact set in $\mathcal{D}_{\uparrow}$ which denotes the Cartesian product $\mathcal{D}_{1} \times \ldots \times \mathcal{D}_{N}$.

We see that $\Phi\left(x_{\uparrow}, \Pi_{\uparrow}\right):=\left(\Phi_{1}\left(x_{1}, \Pi_{1}\right), \ldots, \Phi_{N}\left(x_{N}, \Pi_{N}\right), \Phi_{N+1}\left(x_{\uparrow}\right)\right)$ defines a set mapping from the nonempty compact convex set $X_{\uparrow} \times \mathcal{D}_{\uparrow}$ to nonempty convex compact subsets of $X_{\uparrow} \times \mathcal{D}_{\uparrow}$. If the graph of this set mapping is closed, then Kakutani's fixed point theorem, reproduced in the appendix as Theorem 13, gives a fixed point $\left(\bar{x}_{\uparrow}, \bar{\Pi}_{\uparrow}\right)$. That is, $\bar{x}_{\uparrow}$ solves the Nash game $(23)$ and $\bar{\Pi}_{\uparrow}$ is a marginal risk equilibrium for $\left(\Xi_{i}\left(\bar{x}_{i}, \bar{x}_{-i}\right)\right)_{i}$. In this case, Theorem 11 completes the proof.

To show that the graph of $\Phi$ is closed, suppose $\left\{\left(\hat{x}_{\uparrow}^{k}, \hat{\Pi}_{\uparrow}^{k}\right)\right\}$ is a sequence in $X_{\uparrow} \times \mathcal{D}_{\uparrow}$ that converges to $\left(\hat{x}_{\uparrow}, \hat{\Pi}_{\uparrow}\right)$; write $\hat{Z}_{\uparrow}^{k}=\left(\Xi_{i}\left(\hat{x}_{i}^{k}, \hat{x}_{-i}^{k}\right)\right)_{i}$ and $\hat{Z}_{\uparrow}=\left(\Xi_{i}\left(\hat{x}_{i}, \hat{x}_{-i}\right)\right)_{i}$ so that $\hat{Z}_{\uparrow}^{k} \rightarrow \hat{Z}_{\uparrow}$. Suppose further that $\left(\check{x}_{\uparrow}^{k}, \check{\Pi}_{\uparrow}^{k}\right) \in \Phi\left(\hat{x}_{\uparrow}^{k}, \hat{\Pi}_{\uparrow}^{k}\right)$ converges to $\left(\check{x}_{\uparrow}, \check{\Pi}_{\uparrow}^{k}\right)$ as $k \rightarrow \infty$. Since $\mathbb{E}_{\Pi_{i}}\left[\Xi_{i}\left(x_{i}, x_{-i}\right)\right]$ is continuous in $\left(x_{\uparrow}, \Pi_{i}\right)$, it follows that $\check{x}_{i}$ solves $(23)$ when $\Pi_{i}=\hat{\Pi}_{i}$ and $x_{-i}=\hat{x}_{-i}$. It is only left to show that $\check{\Pi}_{\uparrow}$ is a marginal risk equilibrium for $\hat{Z}_{\uparrow}$.

From Theorem 9, statement 3, there exist $\check{W}_{\uparrow}^{k}$ and $\check{P}^{\text {rk }}$ that satisfy (24) for $Z_{\uparrow}=$ $\hat{Z}_{\uparrow}^{k}$ and $\Pi_{\uparrow}=\check{\Pi}_{\uparrow}^{k}$. Since $\check{W}_{\uparrow}^{k}$ is a solution of the inf convolution problem in statement 2 of Theorem 9 , for $Z_{\uparrow}=Z_{\uparrow}^{k}$, the assumption $\operatorname{int}_{\mathcal{P}}\left(\mathcal{D}_{0}\right) \neq \emptyset$ means that $\left\{\check{W}_{\uparrow}^{k}\right\}$ inherits boundedness from $\left\{\hat{Z}_{\uparrow}^{k}\right\}$. Since $\mathcal{P}$ is bounded, so are $\left\{\check{\Pi}_{\uparrow}^{k}\right\}$ and $\left\{\check{P}^{\mathrm{rk}}=\left.\check{\Pi}_{i}^{k}\right|_{\mathcal{W}}\right\}$. Thus $\left\{\left(\check{W}_{\uparrow}^{k}, \check{P}^{\mathrm{rk}}, \check{\Pi}_{\uparrow}^{k}\right)\right\}$ has a limit point $\left(\check{W}_{\uparrow}, \check{P}^{\mathrm{r}}, \check{\Pi}_{\uparrow}\right)$. Since $\check{\Pi}_{i}^{k} \in \partial r_{i}\left(\hat{Z}_{i}^{k}-\check{W}_{i}^{k}\right)$, then, in the limit, $\check{\Pi}_{i} \in \partial r_{i}\left(\hat{Z}_{i}-\check{W}_{i}\right)$ for each $i$. The balance equation $\sum \check{W}_{i}^{k}=0$ also holds in the limit, as does the identity $\check{P}^{\text {rk }}=\left.\check{\Pi}_{i}^{k}\right|_{\mathcal{W}}$, that is, $\sum \check{W}_{i}=0$ and $\check{P}^{\mathrm{r}}=\left.\check{\Pi}_{i}\right|_{\mathcal{W}}$. 
By Theorem 9 again, $\check{\Pi}_{\uparrow}$ is a marginal risk equilibrium for $\hat{Z}_{\uparrow}$, and the proof is complete.

Note that since the proof assumes existence of strong limit points of bounded sequences in $\mathcal{Z} \times \mathcal{Z}^{*} \times \mathcal{Z}^{*}$, it only covers the case $\mathcal{Z}=\mathbb{R}^{K}$.

\section{Appendix A.}

Proof of Theorem 6. We start with some minor results of convex analysis that we state without proof.

Lemma 1. Let $\mathcal{X}$ be a Banach space with topological dual $\mathcal{X}^{*}$ and $f: \mathcal{X} \rightarrow \mathbb{R}_{\infty}$ be convex with domain $D=\{x: f(x)<\infty\}$. Let $\bar{x} \in D$.

1. For any $x \in \mathbb{R}^{n}, f^{\prime}(\bar{x} ; x)$ exists as an extended value in $[-\infty,+\infty]$ such that $f^{\prime}(\bar{x} ; \cdot)$ is positively homogeneous and sublinear.

2. If $f$ is subdifferentiable at $\bar{x}$, then the support function $\sigma_{\partial f(\bar{x})}$ is the lower semicontinuous hull of $f^{\prime}(\bar{x} ; \cdot)$. In particular, $f^{\prime}(\bar{x} ; x)=\sigma_{\partial f(\bar{x})}(x) \in \mathbb{R}$ for $x \in \operatorname{rint}\left(\operatorname{dom} \sigma_{\partial f(\bar{x})}\right)$, while $f^{\prime}(\bar{x} ; x)=\infty$ for $x \notin \operatorname{dom} \sigma_{\partial f(\bar{x})}$.

3. Let $S$ be a nonempty set in $\mathcal{X}^{*}$ that is convex but not necessarily closed, $K$ be a closed convex cone in $\mathcal{X}$ with nonempty relative interior, and $K^{\circ}:=$ $\left\{\xi \in \mathcal{X}^{*}: \xi[x] \leq 0\right.$ for all $\left.x \in K\right\}$. If $f^{\prime}(\bar{x} ; x) \leq \sigma_{S}(x)$ for $x \in \operatorname{rint} K$, then $\partial f(\bar{x}) \subset \operatorname{cl}\left(S+K^{\circ}\right)$. If $f^{\prime}(\bar{x} ; x)=\sigma_{S}(x) \in \mathbb{R}$ for $x \in \operatorname{rint} K$ and $f^{\prime}(\bar{x} ; x)=\infty$ for $x \notin K$, then $\partial f(\bar{x})=\operatorname{cl}\left(S+K^{\circ}\right)$.

Parts 1 and 2 are directly given by [30, Theorem 11 and prior discussion]. Part 3 follows from the previous parts using the standard facts of convex analysis.

Proof of Theorem 6. If $\mathcal{Z}=\mathbb{R}^{K}$, then standard convex analysis [29] gives the results. For the remainder of the proof let $\mathcal{Z}=L_{p}(\Omega, \mathcal{F}, \mu)$, where $1 \leq p<\infty$. Convexity of $\rho(x, W)$ is an easy extension of [33, Proposition 6.7] to account for possibly infinite values.

Take any $x \in \operatorname{rint} T_{D}(\bar{x})$ and $W \in \mathcal{Z}$. Since there exists $\Xi^{\prime}(\bar{x} ; x) \in \mathcal{Z}$, then there also exists the directional derivative of $\rho$ at $(\bar{x}, \bar{W})$ in the direction $(x, W)$, denoted $\psi(x, W)$, that satisfies

$$
\begin{aligned}
\psi(x, W) & =r^{\prime}\left(\bar{Z} ; \Xi^{\prime}(\bar{x} ; x)-W\right) \\
& =\sup _{\Pi \in \partial r(\bar{Z})} \mathbb{E}_{\Pi}\left[\Xi^{\prime}(\bar{x} ; x)-W\right] \in \mathbb{R},
\end{aligned}
$$

where the first equality is built on the facts that $r$ is Hadamard directionally differentiable (e.g., $[33,(6.44)]$ ) and $\Xi$ is directionally differentiable; the second uses the standard fact that $r^{\prime}(\bar{Z} ; \cdot)$ is the support function of $\partial r(\bar{Z})$ (Lemma 1, part 2) because the latter is real valued (due to boundedness of $\partial r(\bar{Z})$ which relies on continuity of $r$ ); and membership in $\mathbb{R}$ immediately follows.

Take any $\Pi \in \mathcal{P}$. Applying the above discussion of $\rho$ and its directional derivative $\psi$ to the particular case of $r=\mathbb{E}_{\Pi}$, where $\mathbb{E}_{\Pi} \circ \Xi$ takes the value $\infty$ for $Z \notin D$, gives

$$
\mathbb{E}_{\Pi}\left[\Xi^{\prime}(\bar{x} ; x)\right]=\left(\mathbb{E}_{\Pi} \circ \Xi\right)^{\prime}(\bar{x} ; x)=\sigma_{\partial\left(\mathbb{E}_{\Pi} \circ \Xi\right)(\bar{x})}(x) \in \mathbb{R} \quad \text { for } x \in \operatorname{rint} T_{D}(\bar{x}) .
$$

We can also apply $[33$, Theorem 7.47$]$ to $\mathbb{E}_{\Pi} \circ \Xi$ to get

$$
\partial\left(\mathbb{E}_{\Pi} \circ \Xi\right)(\bar{x})=\mathbb{E}_{\Pi}[\partial \Xi(\bar{x})]+N_{D}(\bar{x}),
$$

where $\mathbb{E}_{\Pi}[\partial \Xi(\bar{x})]$ is defined by $(15)$ and $N_{D}(\bar{x}):=\left\{v \in \mathbb{R}^{n}: v^{\top}(x-\bar{x}) \leq 0\right.$ for all $x \in D\}$ is the normal cone to $D$ at $\bar{x}$, and, moreover, that $\mathbb{E}_{\Pi}\left[\Xi^{\prime}(\bar{x} ; x)\right]=$ $\int \Pi_{\omega} \Xi_{\omega}^{\prime}(\bar{x} ; x) d \mu(\omega)$, which justifies the identity $\Xi^{\prime}(\bar{x} ; x)_{\omega}=\Xi_{\omega}^{\prime}(\bar{x} ; x)$ a.e. 
Let $K=T_{D}(\bar{x}) \times \mathcal{Z}$, so that its polar is $K^{\circ}=N_{D}(\bar{x}) \times\{0\}$, and

$$
S=\bigcup_{\Pi \in \partial r(\bar{Z})}\left(\mathbb{E}_{\Pi}[\partial \Xi(\bar{x})] \times\{-\Pi\}\right) .
$$

Combining (25) and (26) with the above characterization of $\psi(x, W)$ yields

$$
\psi(x, W)=\sigma_{S}(x, W) \in \mathbb{R} \quad \text { for }(x, W) \in \operatorname{rint} K .
$$

It is straightforward to show convexity of the union of $\mathbb{E}_{\Pi}[\partial \Xi(\bar{x})]$ over $\Pi \in \partial r(\bar{Z})$, and hence convexity of $S$, using convexity of $\partial r(\bar{x})$ and each $\partial \Xi_{\omega}(\bar{x})$. It is also clear, from first principles, that $\psi(x, W)=\infty$ for $(x, W) \notin K$. So part 3 of Lemma 1 gives $\partial \rho(\bar{x}, \bar{W})=\operatorname{cl}\left(S+K^{\circ}\right)$.

Next we show that $S+K^{\circ}$ is closed. Since it is convex, we will show weak* closure. Take an element $(u, \zeta)$ of $\mathrm{w}^{*}-\mathrm{cl}\left(S+K^{\circ}\right)$, i.e., $\zeta$ is the weak ${ }^{*}$ limit of a net $\left\{-\Pi_{\nu}\right\}$ where $\Pi_{\nu} \in \partial r(\bar{Z})$ for each index $\nu$, and $u=\lim \mathbb{E}_{\Pi_{\nu}}\left[\xi_{\nu}\right]+w_{\nu}$ for some nets $\left\{\xi_{\nu}\right\}$, where $\mathbb{E}_{\Pi_{\nu}}\left[\xi_{\nu}\right] \in \mathbb{E}_{\Pi_{\nu}}[\partial \Xi(\bar{x})]$ for each $\nu$, and $\left\{w_{\nu}\right\} \subset N_{D}(\bar{x})$. Hence $\left\{\Pi_{\nu}\right\}$ weak $^{*}$ converges to $-\zeta$ and, since $\partial r(\bar{Z})$ is weak ${ }^{*}$ closed, its weak ${ }^{*}$ limit is some $\Pi \in \partial r(\bar{Z})$, thus $\zeta=-\Pi$. Take $x \in \operatorname{rint} T_{D}(\bar{x})$ as above. Of course $w_{\nu}^{\top} x \leq 0$, which gives the first inequality in the following chain:

$$
\begin{aligned}
u^{\top} x & \leq \lim _{\nu}\left(\left(\mathbb{E}_{\Pi_{\nu}}\left[\xi_{\nu}\right]\right)^{\top} x\right) \\
& =\lim _{\nu}\left(\mathbb{E}_{\Pi_{\nu}}\left[\left(\xi_{\nu \omega}{ }^{\top} x\right)_{\omega}\right]\right) \\
& \leq \limsup _{\nu} \mathbb{E}_{\Pi_{\nu}}\left[\Xi^{\prime}(\bar{x} ; x)\right] \\
& =\mathbb{E}_{\Pi}\left[\Xi^{\prime}(\bar{x} ; x)\right]=\sigma_{\mathbb{E}_{\Pi}[\partial \Xi(\bar{x})]}(x),
\end{aligned}
$$

where (27) moves $x$ inside the integral that defines $\mathbb{E}_{\Pi_{\nu}}\left[\xi_{\nu}\right]$; the inequality (28) relies a.e. on having $\Pi_{\nu \omega} \geq 0$, the inequality $\xi_{\omega \nu}{ }^{\top} x \leq \sigma_{\partial \Xi_{\omega}(\bar{x})}(x)$ (since $\xi_{\nu \omega} \in \partial \Xi_{\omega}(\bar{x})$ ), and $\sigma_{\partial \Xi_{\omega}(\bar{x})}(x)=\Xi_{\omega}^{\prime}(\bar{x} ; x)$ (Lemma 1, part 2); and in (29), the first equality is due to weak ${ }^{*}$ convergence of $\left\{\Pi_{\nu}\right\}$, while the final inequality is from (25). Lemma 1, part 3, with $K=T_{D}(\bar{x})$ gives $u \in \operatorname{cl}\left(\mathbb{E}_{\Pi}[\partial \Xi(\bar{x})]+N_{D}(\bar{x})\right)$. But $\mathbb{E}_{\Pi}[\partial \Xi(\bar{x})]+N_{D}(\bar{x})$ is already closed because it is the subdifferential of the convex function $\mathbb{E}_{\Pi} \circ \Xi$ at $\bar{x}$ as given by (26). Thus $(u, \zeta) \in S+\left(N_{D}(\bar{x}) \times\{0\}\right)$ as needed.

So $\partial \rho(\bar{x}, \bar{W})=S+\left(N_{D}(\bar{x}) \times\{0\}\right)$ and it only remains to show that $S+\left(N_{D}(\bar{x}) \times\right.$ $\{0\})=S$. Let $\Pi \in \mathcal{P}$. We have $\mathbb{E}_{\Pi}[\partial \Xi(\bar{x})]+N_{D}(\bar{x}) \supset \mathbb{E}_{\Pi}[\partial \Xi(\bar{x})]$ since $0 \in N_{D}(\bar{x})$ and will show that the reverse inclusion also holds. Choose any $\mathbb{E}_{\Pi}[\xi] \in \mathbb{E}_{\Pi}[\partial \Xi(x)]$ and $v \in N_{D}(\bar{x})$. For each $\omega$ define $\xi_{\omega}^{\prime}=\xi_{\omega}+v \in \partial \Xi_{\omega}(\bar{x})+N_{D}(\bar{x})$. For a.a. $\omega$, since $\Xi_{\omega}$ takes the value $\infty$ outside $D$, it is clear from first principles that $\partial \Xi_{\omega}(\bar{x})+N_{D}(\bar{x}) \subset$ $\partial \Xi_{\omega}(\bar{x})$, hence $\xi_{\omega}^{\prime} \in \partial \Xi_{\omega}(\bar{x})$. Moreover $\mathbb{E}_{\Pi}\left[\xi^{\prime}\right]=\mathbb{E}_{\Pi}[\xi]+v$, which shows integrability of $\left(\Pi_{\omega} \xi_{\omega}^{\prime}\right)_{\omega}$, i.e., $\mathbb{E}_{\Pi}[\xi]+v=\mathbb{E}_{\Pi}\left[\xi^{\prime}\right] \in \mathbb{E}_{\Pi}[\partial \Xi(\bar{x})]$. Therefore $\mathbb{E}_{\Pi}[\partial \Xi(\bar{x})]+N_{D}(\bar{x})=$ $\mathbb{E}_{\Pi}[\partial \Xi(\bar{x})]$ and we are done.

Kakutani's and Nash's theorems. Combining the usual norm topology on $\mathbb{R}^{n N}$ with the weak* topology on $\mathcal{Z}^{*}$ will allow us to apply the following infinite dimensional fixed point theorem to the Hausdorff locally convex topological vector space $\mathbb{R}^{n N} \times \mathcal{Z}^{*}$.

TheOREM 13 (Kakutani). Let $\mathcal{C}$ be a nonempty, convex, compact set in a Hausdorff locally convex topological vector space, and let $\Psi: \mathcal{C} \rightarrow \mathcal{C}$ be a set-valued mapping. If $\Psi(c)$ is a nonempty and convex set (in $\mathcal{C}$ ) for all $c \in \mathcal{C}$, and the graph 
$\{(c, d): c \in \mathcal{C}, d \in \Psi(c)\}$ is closed, then $\Psi$ has a fixed point: there exists $c^{*} \in \mathcal{C}$ such that $c^{*} \in \Psi\left(c^{*}\right)$.

Theorem 13 is obtained from [19, p. 186], in which the closed graph property is replaced by upper semicontinuity, by noting that in Hausdorff (or separated) topological spaces, a set-valued mapping is upper semicontinuous if (and only if) it has a closed graph.

Given Theorem 13 we state the corresponding infinite dimensional version of Nash's theorem [24] whose proof is identical to the original. We specify topological properties of the objective function $f_{i}$ that were not mentioned in the original, however.

Theorem 14 (Nash). For $i=1, \ldots, N$, let $f_{i}: S_{i} \times S_{-i} \rightarrow \mathbb{R}$, where $S_{i}$ is a set in a Hausdorff locally convex topological vector space $\mathcal{S}_{i}$ and $S_{-i}$ denotes the Cartesian product of sets $S_{j}$ for $j \neq i, j=1, \ldots, N$. Similarly, let $s_{-i}$ denote the stacked vector consisting of $s_{j}$ for $j \neq i$.

Consider the (noncooperative pure strategy) Nash game. Each agent $i$ is given others' strategies $s_{-i}$ and wishes to solve $\min _{s_{i} \in S_{i}} f_{i}\left(s_{i}, s_{-i}\right)$. Suppose each $S_{i}$ is a nonempty convex compact set; the lower level set $\left\{s_{i} \in S_{i}: f_{i}\left(s_{i}, s_{-i}\right) \leq \alpha\right\}$ is convex for each $s_{-i} \in S_{-i}$ and scalar $\alpha ; f_{i}\left(s_{i}, \cdot\right)$ is continuous on $S_{-i}$ given $s_{i} \in S_{i}$, and $f_{i}$ is lower semicontinuous on $S_{i} \times S_{-i}$. Then there is a Nash equilibrium $\left(s_{1}^{*}, \ldots, s_{N}^{*}\right)$ : each $s_{i}^{*}$ solves $\min _{s_{i} \in S_{i}} f_{i}\left(s_{i}, s_{-i}^{*}\right)$.

Acknowledgments. We thank Andrew Eberhard, Andreas Ehrenmann, Nicolas Grebille, Jong-Shi Pang, Andy Philpott, Eric Ralph, Andrzej Ruszczyński, and Nicos Savva, whose comments have improved the paper. We also thank anonymous referees, one of whom supplied several references that allowed us to significantly condense the material on risk markets.

\section{REFERENCES}

[1] K. J. ARrow, The role of securities in the optimal allocation of risk-bearing, Rev. Econom. Stud., 31 (1964), pp. 91-96.

[2] P. Artzner, F. Delbaen, J. M. Eber, and D. Heath, Coherent measures of risk, Math. Finance, 9 (1999), pp. 203-228.

[3] H. Attouch And H. BrÉZIs, Duality for the sum of convex functions in general Banach spaces, in Aspects of Mathematics and Its Applications, J. Barroso, ed., Elsevier Science Publications, New York, 1986, pp. 125-133.

[4] P. BARRIEU AND N. El KAROUI, INF-convolution of risk measures and optimal risk transfer, Finance Stoch., 9 (2005), pp. 269-298.

[5] A. Ben-Tal and M. Teboulle, Expected utility, penalty functions, and duality in stochastic nonlinear programming, Management Sci., 32 (1986), pp. 1445-1466.

[6] A. Ben-Tal and M. Teboulle, An old-new concept of convex risk measures: The optimized certainty equivalent, Math. Finance, 17 (2007), pp. 449-476.

[7] C. Burgert And L. Rüschendorf, Allocations of risks and equilibrium in markets with finitely many traders, Insurance Math. Econom., 42 (2008), pp. 177-188.

[8] A. S. Cherny, Equilibrium with Coherent Risk, arXiv:math/0605051v1 [math.PR], 2006.

[9] J. H. Cochrane and J. SÁA-Requejo, Beyond arbitrage: Good deal asset price bounds in incomplete markets, J. Political Economy, 108 (2000), pp. 79-119.

[10] A. D. Dana And C. Le VAn, Overlapping sets of priors and the existence of efficient allocations and equilibria for risk measures, Math. Finance, 20 (2010), pp. 327-339.

[11] A. Ehrenmann and Y. Smeers, Generation capacity expansion in a risky environment: A stochastic equilibrium analysis, Oper. Res., 59 (2011), pp. 1332-1346.

[12] A. Ehrenmann And Y. Smeers, Stochastic equilibrium models for generation capacity expansion, in Stochastic Optimization Methods in Finance and Energy, M. Bertocchi, G. Consigli, and M. A. H. Dempster, eds., Springer, New York, 2011, pp. 273-311.

Copyright $@$ by SIAM. Unauthorized reproduction of this article is prohibited. 
[13] F. FACChinei And J. S. PAng, Finite-Dimensional Variational Inequalities and Complementarity Problems, Vol. 1, Springer-Verlag, New York, 2003.

[14] D. Filipović And M. Kupper, Equilibrium prices for monetary utility functions, Int. J. Theor. Appl. Finance, 11 (2008), pp. 325-343.

[15] D. Filipović And G. Svindland, Optimal capital and risk allocations for law- and cashinvariant convex functions, Finance Stoch., 12 (2008), pp. 423-439.

[16] H. Föllmer And A. Schied, Convex measures of risk and trading constraints, Finance Stoch., 6 (2002), pp. 429-447.

[17] P. T. HARker And J. S. PANG, Finite-dimensional variational inequality and nonlinear complementarity problems: A survey of theory, algorithms and applications, Math. Program., 48 (1990), pp. 161-220.

[18] D. Heath and H. Ku, Pareto equilibria with coherent measures of risk, Math. Finance, 14 (2004), pp. 163-172.

[19] R. B. Holmes, Geometric Functional Analysis and Its Applications, Springer-Verlag, New York, 1975.

[20] H. Jiang, S. Netessine, And S. Savin, Technical note: Robust newsvendor competition under asymmetric information, Oper. Res., 51 (2011), pp. $254-261$.

[21] A. Jofré, R. T. Rockafellar, And R. J.-B. Wets, Convex Analysis and Financial Equilibrium, manuscript, https://www.math.ucdavis.edu/ rjbw/mypage/Equilibirum_files/ JfRW13_fincial.pdf (2013).

[22] G. De Maere dAertrycke and Y. Smeers, Liquidity risks on power exchanges, Math. Program., 140 (2013), pp. 381-414.

[23] M. Magill and M. QuinziI, Incomplete Markets. Volume 1: Finite Horizon Economies, International Library of Critical Writings in Economics Series, Edward Elgar Publishing, Cheltenham, UK, 2008.

[24] J. F. NASH, Equilibrium points in n-person games, Proc. Natl. Acad. Sci. USA, 36 (1950), pp. $48-49$.

[25] A. Philpott, M. Ferris, and R. Wets, Uncertainty and risk in hydro-thermal electricity systems, Math. Program., to appear.

[26] D. Ralph and Y. Smeers, Pricing Risk under Risk Measures: An Introduction to StochasticEndogenous Equilibria, working paper, http://papers.ssrn.com/sol3/papers.cfm?abstract_ $\mathrm{id}=1903897$ (2011).

[27] U. V. Ravat and U. V. Shanbhag, On the Existence of Solutions to Stochastic QuasiVariational Inequality and Complementarity Problems, working paper, 2014.

[28] W. J. Robertson And A. P. Robertson, Topological Vector Spaces, Cambridge University Press, Cambridge, UK, 1973.

[29] R. T. Rockafellar, Convex Analysis, Princeton University Press, Princeton, NJ, 1970.

[30] R. T. Rockafellar, Conjugate Duality and Optimization, SIAM, Philadelphia, 1974.

[31] A. Ruszczyński And A. Shapiro, Optimization of convex risk functions, Math. Oper. Res., 31 (2006), pp. 433-452.

[32] P. A. Samuelson, Foundations of Economic Analysis, Harvard University Press, Cambridge, MA, 1947.

[33] A. Shapiro, D. Dentcheva, and A. Ruszczyński, Lectures on Stochastic Programming: Modeling and Theory, SIAM, Philadelphia, 2009.

Copyright $@$ by SIAM. Unauthorized reproduction of this article is prohibited. 